\title{
Mesangial Cells and Glomerular Endothelial Cells Crosstalk in Rat Anti-Thy-1 Nephritis through VEGFA/VEGFAR2 and Angpt2/Tie2 pathway
}

yinghua zhao

Jilin University https://orcid.org/0000-0003-2101-392X

Bo Fu

Chinese PLA General Hospital

Pu Chen

Chinese PLA General Hospital

Qinggang Li

Chinese PLA General Hospital

Qing Ouyang

Chinese PLA General Hospital

Chuyue Zhang

Chinese PLA General Hospital

Guangyan Cai

Chinese PLA General Hospital

Lingling Wu

Chinese PLA General Hospital

Xiangmei Chen ( $\nabla$ xmchen301@126.com )

Chinese PLA General Hospital

\section{Research}

Keywords: mesangial proliferative glomerulonephritis, mesangial cells, glomerular endothelial cells, VEGFA/VEGFR2 pathway, Angpt2/Tie2 pathway

Posted Date: October 28th, 2020

DOI: https://doi.org/10.21203/rs.3.rs-96698/v1

License: (1) (1) This work is licensed under a Creative Commons Attribution 4.0 International License.

Read Full License 


\section{Abstract}

\section{BACKGROUND}

Mesangial proliferative glomerulonephritis is characterized by the proliferation of mesangial cells (MCs). Endothelial cells (ECs) are affected by signals from MCs, resulting in capillary proliferation, but the specific signaling pathway associated with this activity remains unclear.

\section{RESULTS}

In this study, the expression of PCNA, RECA-1 and CD34 in the glomeruli increased on the $7^{\text {th }}$ day after anti-Thy-1 nephritis establishment, indicating the occurrence of ECs proliferation. After coculturing MCs and ECs in vitro, we observed that activated MCs could promote ECs proliferation, migration and a-SMA expression. Moreover, activated ECs had the same effects on MCs. RT-qPCR showed that activated MCs could increasingly secrete VEGFA, and Angpt2 expression in VEGFA-activated ECs was enhanced. Considering that Angpt2-mediated inhibition of ECs surface receptor Tie2 phosphorylation causes ECs proliferation, we hypothesized that VEGFA/VEGFR2 and Angpt2/Tie2 signaling is involved in the interaction between MCs and ECs. Our results showed that blocking VEGFA or adding the Angpt2 antagonist Angpt 1 to the coculture system decreased the number of EdU-positive cells $₫$ Angpt2खp-VEGFR2 and p-MAPK expression, but increased p-Tie2 in ECs. To determine whether Angpt1 could effectively alleviate the pathological changes of anti-Thy-1 nephritis, we performed Vasculotide (Angpt1 mimic peptide) treatment assays in vivo. The results confirmed that the addition of Vasculotide could effectively reduce PCNA, RECA-1 and a-SMA expression and promote $p$-Tie2.

\section{CONCLUSION}

In summary, the study showed that the VEGFA/VEGFR2 and Angpt2/Tie2 signaling pathway mediate interactions between MCs and ECs, providing an important theoretical basis for the treatment of mesangial proliferative glomerulonephritis.

\section{Background}

The main pathological changes in mesangial proliferative glomerulonephritis are mesangial cells (MCs) proliferation and increased extracellular matrix deposition [1-4]. Abnormally proliferating MCs produce inflammatory factors, which lead to fibrosis and glomerular sclerosis and eventually result in end-stage renal disease (ESRD)[5]. Endothelial cells (ECs), another inherent cell in the kidney, play an important role in the integrity of the glomerular vascular bed[6]. Current studies have shown that damage to glomerular ECs appears in both acute and chronic kidney disease with mesangial proliferative glomerulonephritis [7, 8]. As the most suitable animal model of mesangial proliferative glomerulonephritis, the rat anti-Thy-1 nephritis model is often used to study the specific pathogenesis of mesangial proliferative glomerulonephritis $[5,9]$. The staining results of pathological mesangial proliferative glomerulonephritis tissue showed that when MCs were lysed, glomerular capillaries proliferated diffusely and developed 
aneurysmal changes[10]. As MCs proliferated and the mesangial matrix increased, the "aneurysm" gradually decreased until the glomeruli returned to normal[11, 12]. MCs are adjacent to ECs, so instead of communicating directly through gap junctions, both cell types can produce cytokines through paracrine signaling[13]. Interactions between MCs and ECs play an important role in the pathological changes in mesangial proliferative glomerulonephritis. However, there have been few studies on this topic. Therefore, we aimed to explore the specific signaling pathway between MCs and ECs to further understand the pathogenesis of mesangial proliferative glomerulonephritis and provide new ideas for disease treatment.

Under physiological conditions, vascular endothelial growth factor (VEGF) is mainly produced by podocytes, distal tubules and collecting duct epithelial cells[14]. VEGFA, an endothelial mitogen, binding to the ECs surface receptor VEGFR2, plays an important role in promoting angiogenesis[15]. Tie2, also known as TEK in humans, is expressed on the surface of ECs and is a tyrosine kinase receptor with two homologous domains, IG and EGF[16]. Angiopoietin 1 (Angpt1) and Angpt2, two Tie2 ligands, bind to the same domain. Therefore, these ligands competitively bind to Tie2, which is important in angiogenesis and vascular inflammation. Angpt 1 binds to Tie2 to promote Tie2 phosphorylation, thereby maintaining vascular structural integrity, protecting ECs from apoptosis, and inhibiting the inflammatory response[1719]. Angpt2 is expressed and stored in the Weibel-Palade body of ECs. When ECs are stimulated by inflammatory factors (VEGF or TNF and various environmental signals (hypoxia, oxidative stress, and high glucose), a large amount of Angpt2 is released, which leads to decreased ECs stability and promotes vascular proliferation by interfering with the Angpt1/Tie2 signaling pathway[20]. Martina Schmittnaegel et al. pointed out that bispecial-antibody (A2V), which blocked both VEGFA and Angpt2, has a better therapeutic effect than single antibody on metastatic breast cancer, pancreatic neuroendocrine tumor, and melanoma, indicating that VEGFA and Angpt2 have synergistic effects in proangiogenesis[21]. VEGFA or Angpt2/Tie2 signaling pathways is involved in the development and progression of various kidney diseases[22, 23]. However, few studies have investigated the role of the VEGFA/VEGFR2 and Angpt2/Tie2 signaling pathway in mesangial proliferative glomerulonephritis. In summary, we established an in vitro coculture system to explore whether VEGFA/VEGFR2 and Angpt2/Tie2 mediate MCs and ECs interactions to further clarify the pathogenesis of mesangial proliferative glomerulonephritis.

Angpt1 or Angpt1 mimic peptide is used to treat a variety of vascular injury-related diseases, including diseases of the cardiovascular, respiratory and nervous systems[24-27]. However, in the field of kidney disease, the application of Angpt1 is limited, and even fewer studies on mesangial proliferative glomerulonephritis have been performed. In this study, Vasculotide (Angpt1 mimic peptide)[28] was administered to rat anti-Thy- 1 nephritis model for the first time to determine whether it could alleviate the pathological changes in mesangial proliferative glomerulonephritis.

\section{Results}




\section{ECs proliferation was observed in the glomeruli of anti-Thy- 1 nephritis rats}

The rat anti-Thy-1 nephritis model was successfully established, according to that urinary albumin/creatinine ratio was significantly higher on the 5 th and 7 th day (Fig. $1 \mathrm{~d}$. control vs model. 3day: $113.1 \pm 40.33$ vs $379.0 \pm 167.5$, no statistical significance; 5 day: $119.3 \pm 74.14$ vs $466.0 \pm 131.6, p<0.5$; 7day: $182.7 \pm 41.55$ vs $547.0 \pm 185.4, p<0.5$ ). PAS staining results showed that the number of cells in the glomeruli increased significantly on the 7th day after the model was established (Fig. 1a and 1c. control vs model. $47.76 \pm 4.142$ vs $66.36 \pm 4.521, \mathrm{p}<0.01)$. Moreover, RECA-1 and PCNA immunohistochemical staining and immunofluorescence co-staining results showed that the number of glomerular ECs increased (Fig. 1a,1b and 1e. control vs model. RECA-1: $7.616 \pm 1.68$ vs $17.23 \pm 2.694, p<0.01$; PCNA: $0.078 \pm 0.015$ vs 0.353 vs $0.059, p<0.01$ ). Western blot results showed that expression of CD34 in glomeruli was significantly increased in the model group (Fig. $1 \mathrm{f}$ and $1 \mathrm{~g}$. control vs model. $1.0 \pm 0.09 \mathrm{vs}$ $1.922 \pm 0.21, p<0.01)$. These results indicate that there was abnormal ECs proliferation in the model group on the 7 th day after the model was established.

\section{MCs and ECs interactions were confirmed by coculture in vitro}

First, we detected significant increases of PDGF-BB and VEGFA expression on the 7th day of anti-Thy-1 nephritis (Supplementary Figure S2. control vs model. PDGF-BB: $1.0 \pm 0.53$ vs $3.74 \pm 0.74, p<0.01$; VEGFA: $1.0 \pm 0.73$ vs $6.7 \pm 3.44, p<0.5$ ). To reveal the interaction between MCs and ECs, we established an in vitro coculture system. To investigate the effect of activated MCs on ECs proliferation, we cultured MCs in the upper compartment of 24-well Transwell inserts with $0.4 \mu \mathrm{m}$ pores (cytokines but not cells could pass through). First of all, we treated MCs with the classic mesangial activation factor PDGF-BB for $24 \mathrm{~h}$ to imitate the disease state. Then, the upper compartment with activated MCs that cultured in medium without PDGF-BB was attached to the lower chamber with ECs, and the two types of cells were cocultured for $24 \mathrm{~h}$ (Fig. 2a). The EdU results showed that activated MCs significantly promoted the proliferation of ECs (Fig. $2 b$ and $2 d$. control vs PDGF-BB. $0.14 \pm 0.017$ vs $0.34 \pm 0.009, p<0.01$ ). Moreover, immunofluorescence (Fig. $2 c$ and 2e. control vs PDGF-BB. $4.98 \pm 0.78$ vs $8.75 \pm 1.24, p<0.5$ ) and Western blot (Fig. 2 f. control vs PDGF-BB. $1.0 \pm 0.11$ vs $5.35 \pm 0.15, p<0.01$ ) results showed that activated MCs promoted a-SMA expression in ECs. And the upper compartment with ECs was attached to the lower chamber with activated MCs to show that activated MCs promoted ECs migration by using crystal violet staining (Fig. 2g). Thus, activated MCs could enhance the proliferation, a-SMA expression and migration of ECs.

Subsequently, VEGFA-treated ECs were cocultured with MCs for $24 \mathrm{~h}$ (Fig. 3a). EdU (Fig. 3b and 3d. control vs VEGFA. $0.13 \pm 0.04$ vs $0.29 \pm 0.04, p<0.01$ ), a-SMA immunofluorescence (Fig. 3c and 3e. control vs VEGFA. $2.83 \pm 0.78$ vs $9.52 \pm 1.2, p<0.01$ ) and Western blot (Fig. 3 f. control vs VEGFA. $1.0 \pm 0.1$ vs $1.9 \pm 0.09, p<0.01$ ), and crystal violet staining (Fig. $3 g$ ) results confirmed that the VEGFA-activated ECs 
could promote MCs proliferation, a-SMA expression and migration. These results showed that there were obvious interactions between MCs and ECs.

\section{VEGFA/VEGFR2 participates in the interaction between MCs and ECs}

Activated MCs can secrete a variety of cytokines (such as IL-1, IL-6, and TGF- $\beta$ ) that are involved in the development and progression of renal diseases. VEGFA, an ECs-specific mitogen, regulates physiological and pathological angiogenesis. Therefore, we measured the expression of VEGFA in activated MCs to explore whether VEGFA is involved in the interaction between MCs and ECs. The RT-qPCR results showed that the mRNA expression of VEGFA in activated MCs was significantly increased (Fig. 4b. control vs PDGF-BB. $1.0 \pm 0.44$ vs $3.17 \pm 0.19, p<0.01$ ). Then, VEGFA neutralizing antibodies were added to the coculture system (Fig. 4a), and EdU assay results showed that this treatment significantly inhibited activated MCs-mediated ECs proliferation (Fig. $4 \mathrm{c}$ and $4 \mathrm{e}$. control vs PDGF-BB vs PDGF-BB + anti-VEGFA. $0.51 \pm 0.03$ vs $0.86 \pm 0.06$ vs $0.52 \pm 0.1, p<0.01$ ). Western Blots pointed that activated MCs can definitely improve VEGFR2 phosphorylation, which was inhibited by VEGFA neutralizing antibody (Fig. 4d. control vs PDGF-BB vs PDGF-BB + anti-VEGFA. $1.0 \pm 0.1$ vs $1.22 \pm 0.08$ vs $0.8 \pm 0.07, \mathrm{p}<0.01$ ). Thus, activated MCs can generate VEGFA, which binds with VEGFR2 and promotes ECs proliferation.

\section{The Angpt2/Tie2 signaling pathway is involved in MCs and ECs interactions}

Current studies have shown that VEGFA and Angpt2 both alter vascular structure and function and are involved in cardiovascular and tumor-related diseases[30-32]. Angpt2 is expressed by ECs and binds to Tie2 to promote ECs proliferation by inhibiting Tie2 phosphorylation. Thus, we hypothesized that the VEGFA/VEGFR2 and Angpt2/Tie2 signaling pathway play an important role in activated MCs-induced ECs proliferation. We also established a coculture system to confirm this hypothesis and assayed the effect of VEGFA neutralizing antibodies. The RT-qPCR results showed that ECs could express more Angpt2 mRNA when activated by VEGFA or cocultured with activated MCs (Fig. 5a. control vs VEGFA. 1.0 \pm 0.09 vs $1.83 \pm 0.81, p<0.5$. control vs PDGF-BB. $1.0 \pm 0.16$ vs $3.0 \pm 0.76, p<0.5)$. Immunofluorescence and western blot results showed that activated MCs promoted ECs Angpt2 expression (Fig. 5b. control vs PDGF-BB vs PDGF-BB + anti-VEGFA. $0.40 \pm 0.32$ vs $3.02 \pm 1.19$ vs $0.87 \pm 0.18, p<0.5$ ) and inhibited Tie2 phosphorylation (Fig. 5c and 5d. control vs PDGF-BB vs PDGF-BB + anti-VEGFA. $1.0 \pm 0.22$ vs $0.85 \pm 0.03$ vs $1.3 \pm 0.14, p<0.01$ ), which could be abrogated by treatment with VEGFA neutralizing antibodies. Thus, activated MCs, expressing VEGFA, can promote ECs proliferation by promoting Angpt2 expression and inhibiting Tie2 phosphorylation.

\section{Angpt1 can effectively alleviate activated MCs-induced ECs proliferation}


Angpt1 and Angpt2 can bind to the same part of Tie2, but they have opposite effects. The binding of Angpt1 to Tie2 can promote Tie2 phosphorylation, stabilizing ECs and inhibiting vascular proliferation. Therefore, we hypothesized that Angpt1, a competitive inhibitor of Angpt2, could effectively alleviate activated MCs-induced ECs proliferation. Thus, Angpt1 was added to the coculture system (Fig. 6a), and the EdU results showed that Angpt1 could effectively alleviate activated MCs-mediated ECs proliferation (Fig. $6 \mathrm{~b}$ and $6 \mathrm{f}$. control vs PDGF-BB vs PDGF-BB + Angpt1. $0.33 \pm 0.02$ vs $0.54 \pm 0.04$ vs $0.37 \pm 0.07$, $p<$ 0.01). Moreover, immunofluorescence and Western blot results showed that Angpt1 did not alter the expression of Angpt2 in ECs (Fig. $6 \mathrm{c}$ and $6 \mathrm{~g}$. control vs PDGF-BB vs PDGF-BB + anti-VEGFA. $0.56 \pm 0.22$ vs $4.59 \pm 0.84$ vs $4.96 \pm 1.14, p<0.01$ ), while Tie2 phosphorylation was significantly increased (Fig. 6d, 6e and 6i. control vs PDGF-BB vs PDGF-BB + Angpt1. $1.0 \pm 0.07$ vs $0.82 \pm 0.06$ vs $1.06 \pm 0.09, p<0.5)$. Thus, Angpt1 competitively binds to Tie2 and inhibits Angpt2 binding to Tie2, which promotes Tie2 phosphorylation and inhibits cell proliferation. In addition, the study of ECs proliferation showed that the expression of p-MAPK increased when co-cultured with activated MCs, while decreased after adding Angpt1 (Fig. 6h and 6j. control vs PDGF-BB vs PDGF-BB + Angpt1. $1.0 \pm 0.03$ vs $1.35 \pm 0.1$ vs $0.74 \pm 0.06$, $p<0.01)$. Thus, ECs proliferation was promoted through MAPK signaling pathway.

\section{Angpt1 mimic peptide can effectively alleviate cell proliferation in rat anti-Thy-1 nephritis on day 7}

To further explore whether Angpt1 can effectively alleviate cell proliferation in vivo, we established the anti-Thy-1 rat model and administered Angpt1 mimic peptide (Vasculotide). The rats were divided into the control group, model group and Angpt1-treated group. All rats were sacrificed on the 7th day after the model was established (Fig. 7a). PAS staining results showed that the hypercellularity index per glomerulus decreased significantly on the 7th day after the Vasculotide was treated (Fig. 7b and 7d. control vs model vs Angpt1. $0.2 \pm 0.1$ vs $1.7 \pm 0.15$ vs $1.0 \pm 0.21, p<0.01)$. Immunohistochemical results showed decreased a-SMA, RECA-1 and PCNA expression in the Angpt1-treated group (Fig. 7b and 7e. control vs model vs Angpt1. a-SMA: $0.69 \pm 0.4$ vs $13.22 \pm 2.23$ vs $1.32 \pm 0.93, p<0.01$; RECA-1: $10.92 \pm$ 1.44 vs $15.73 \pm 3.63$ vs $10.1 \pm 0.73, p<0.01$; PCNA: $0.08 \pm 0.01$ vs $0.38 \pm 0.04$ vs $0.12 \pm 0.01, p<0.01$ ). And PCNA and RECA-1 immunofluorescence co-staining result showed that the number of double-positive cells in the Angpt1-treated group was decreased compared to that observed in the model group (Fig. 7b). Western blot results showed that the expression of a-SMA and CD34 in the model group was significantly increased, and Tie2 phosphorylation was downregulated. After Vasculotide treatment, the expression of a-SMA and CD34 was decreased, and Tie2 phosphorylation was increased, while the expression of cleaved caspase3 was not affected (Fig. 7c. control vs model vs Angpt1. p-Tie2: $1.0 \pm 0.3$ vs $0.13 \pm 0.12$ vs $0.78 \pm 0.22$, $p<0.01 ;$ CD $34: 1.0 \pm 0.36$ vs $2.68 \pm 0.25$ vs $1.34 \pm 0.37, p<0.01 ; a-S M A: 1.0 \pm 0.86$ vs 3.83 \pm 0.22 vs $1.85 \pm 1.01, p<0.01$; cleaved-caspase $3: 1.0 \pm 0.14$ vs $1.43 \pm 0.12$ vs $1.45 \pm 0.44$, no statistical significance). These results showed that on the 7th day of anti-Thy-1 nephritis, cell proliferation in the glomerulus was obvious and Tie2 phosphorylation was inhibited. Thus, Angpt1 mimic peptide could effectively alleviate ECs proliferation by promoting Tie2 phosphorylation. 


\section{Discussion}

Studies related to mesangial proliferative glomerulonephritis have shown that when MCs injury and mesangial matrix accumulation occur in the kidney, ECs are affected by signals from MCs, presenting pathological changes such as diffuse capillary proliferation[33]. These findings indicate that there is a connection between MCs and ECs, and both cell types participate in mesangial proliferative glomerulonephritis pathogenesis. To investigate the cytokines involved in the interaction between MCs and ECs, we established a coculture system, which showed that PDGF-BB-activated MCs or VEGFAactivated ECs promoted proliferation and migration of the other cell type. Under a disease state, activation of one cell type can activate the other cell type, leading to cell proliferation and migration and forming a vicious cycle. Therefore, we wondered if we could alleviate disease progression by blocking part of the loop. The aim of this study was to explore the specific molecular mechanism of the interaction between MCs and ECs and to provide new ideas for mesangial proliferative glomerulonephritis treatments.

Imbalanced VEGF expression in glomeruli has been demonstrated in a variety of primary and acquired renal diseases[34]. VEGFA plays a key role in maintaining the glomerular filtration barrier by affecting apoptosis, proliferation and differentiation of ECs[35-37]. Numerous studies have demonstrated that significant changes in VEGFA are associated with a variety of kidney diseases. In diabetic nephropathy (DN), VEGFA is involved in a variety of signaling pathways (e.g., VEGFR2/nephrin, VEGF/nephrin, and VEGF/ nitric oxide synthase (eNOS-NO) and is associated with insulin resistance and eNOS and Angpt expression[38]. Urinary VEGFA levels are significantly higher in lupus nephritis patients than in lupus patients without renal damage[39]. To further elucidate whether VEGFA is involved in signal transduction between MCs and ECs, VEGFA neutralizing antibodies were added to the coculture system. The results showed that VEGFA neutralizing antibodies significantly inhibited MCs-induced ECs proliferation. The results of this study not only confirmed the involvement of VEGFA in intercellular interactions but also provided a new idea for the clinical treatment of mesangial proliferative glomerulonephritis; that is, VEGFA neutralizing antibodies can be used to block the effect of injured MCs on ECs to effectively alleviate diffuse ECs proliferation during early MCs lysis. However, there are drawbacks to this approach. Studies on DN and oncology have shown that VEGF-blocking treatment can cause renal injury[14]. Because it is acknowledged that most kidney diseases are associated with severe immune system disorders, VEGFA neutralizing antibodies, which bind to VEGFA, can form antigen-antibody complex deposits in the kidney. Contrary to achieving the desired outcome, VEGFA neutralizing antibodies may exacerbate kidney damage. The glomeruli, on the other hand, require some VEGFA-mediated signaling to function normally. Therefore, VEGFA neutralizing antibodies were not used to treat anti-Thy- 1 nephritis in this study.

Angiopoietins belongs to a family of growth factors and includes Angpt1 and Angpt2. Angpt2 signals through endocrine or autocrine mechanisms and is released by Weibel-Palade bodies in ECs[40]. Angpt2 damages the function of ECs by inhibiting Angpt1 binding to Tie2. In studies on chronic kidney disease (CKD), lupus nephritis, thrombotic microangiopathy (TMA) and anti-glomerular basement membrane 
(GBM) nephropathy, Angpt2 expression was increased in patient serum, while Angpt1 expression was decreased[41-43]. Angpt2 is associated with a variety of diseases (such as diabetes, hypertension, coronary heart disease and CKD) and elevated Angpt2 levels are associated with poor prognosis[44]. Relevant studies have shown that Angpt2 collaborates with VEGFA to promote angiogenesis[45, 46$]$. In breast cancer, non-small cell lung cancer and acute myeloid leukemia, the expression of VEGFA and Angpt2 increased simultaneously, which promoted tumor growth. In addition, in the presence of high VEGFA expression, Angpt2 promotes hepatoma carcinoma cell growth[47]. In this study, VEGFA expression in activated MCs was increased, and VEGFA promoted Angpt2 expression in ECs. Thus, in addition to VEGFA, Angpt2 also plays an important role in signal transduction between MCs and ECs.

Angpt1, another Tie2 ligand, has the same binding site as Angpt2. The Angpt1/Tie2 signaling pathway is highly expressed in mature and stable vessels and can inhibit the increase in vascular osmotic pressure caused by inflammatory factors such as VEGFA[48]. In various experimental kidney disease models, Angpt 1 has a protective effect. Therefore, the Angpt1/Tie2 signaling pathway is a target for the development of antiangiogenic drugs. In this study, the addition of Angpt1 to the coculture system increased Tie2 phosphorylation and inhibited cell proliferation, but Angpt2 expression was not affected. Studies have shown that Angpt 1 can reduce the risk of acute kidney injury (AKI) in critically ill patients [49] and can promote renal recovery by enhancing EC, suggesting that Angpt1 is a potential AKI treatment[50]. Studies have also shown that injecting COMP-Angpt1 (synthetically modified Angpt1) into $\mathrm{db} / \mathrm{db}$ mice can reduce proteinuria and alleviate mesangial expansion and GBM thickening, indicating that Angpt1 is also beneficial in DN[51]. Combined with the results of this study, we believe that Angpt1 can also be used to alleviate mesangial proliferative glomerulonephritis-associated vascular injury. Studies indicate[52] that peak ECs proliferation occurred on the 7th day of anti-Thy-1 nephritis, and so we injected Angpt 1 mimic peptide (Vasculotide) on the 4th, 5th and 6th days after model induction. The results showed that Vasculotide significantly inhibited ECs proliferation on the 7th day. Moreover, Vasculotide did not form antigen-antibody immune complexes and is safer than the VEGFA neutralizing antibody. Furthermore, Vasculotide competitively inhibits the effect of Angpt2, only blocking the downstream VEGFA signaling pathway, without affecting other normal functions.

\section{Conclusion}

In summary, the present study found that the VEGFA/VEGFR2 and Angpt2/Tie2 signaling pathway was involved in the interaction between MCs and ECs. Blocking VEGFA (VEGFA neutralizing antibody) or adding an Angpt2 competitive inhibitor (Angpt1) can inhibit activated MCs-mediated ECs proliferation. Moreover, in vivo experiments showed that Angpt1 mimic peptide (Vasculotide) could alleviate ECs proliferation on the 7th day of anti-Thy-1 nephritis, which provided a new idea and strategy for alleviating the pathological changes in mesangial proliferative glomerulonephritis.

\section{Materials And Methods}

\section{Vasculotide}


Tournaire R. et al. [29] were the first to synthesize a short peptide HHHRHSF whose tetramer forming by affinity/biotin could combine with the extracellular part of Tie2 and play a role similar to Angpt1. At present, 4-arms polyethanol glycol (average molecular weight $10 \mathrm{kDa}$ ) scaffold is used to replace the avidin/biotin complex. In our study, Vasculotide was synthesized by Shanghai Bootech Bioscience \& Technology Co (Supplementary Figure S1).

\section{Animals}

Wild-type Wistar rats (aged 6-8 weeks, 200-250 g) were purchased from the Animal Center of PLA General Hospital. All rats were kept in an environment with constant temperature $\left(20^{\circ} \mathrm{C}\right)$, humidity $(70 \%)$ and alternating day and night cycles. To explore the pathological changes in ECs in anti-Thy- 1 nephritis, the rats were divided into a control group $(n=7)$ and a model group $(n=7)$. An anti-Thy- 1 antibody $(2.5 \mathrm{mg} / \mathrm{kg})$ was administered by tail vein injection to rats in the model group to establish rat anti-Thy-1 nephritis. The control group was injected with saline solution. To evaluate the role of Angpt1 mimic peptide (Vasculotide) in anti-Thy- 1 nephritis, we divided the rats into a control group $(n=7)$, model group $(n=7)$ and Angpt1 group $(n=11)$. The Angpt1 intervention group was repeatedly injected with Angpt1 mimic peptide (Vasculotide, $3 \mu \mathrm{g} / \mathrm{kg}$, i.p.) on the 4th, 5th and 6th days. On the 7th day, the rats were sacrificed. To isolate the glomeruli, the rat kidneys were washed with cold saline, and the renal capsule was removed. The renal cortex was cut into fragments and filtered through $250 \mu \mathrm{m}, 106 \mu \mathrm{m}$ and $75 \mu \mathrm{m}$ sieves. Glomeruli were collected on the upper layer of the $75 \mu \mathrm{m}$ sieve and were observed under the microscope. Glomeruli collection was successful when renal tubular cells were barely observed (glomerulus purity $>95 \%$ ). The glomeruli were then washed and transferred to centrifuge tubes. After centrifugation, the supernatant was discarded, and the separated glomerular precipitate was stored at $-80^{\circ} \mathrm{C}$. The experiments were performed according to the Guide for the Care and Use of Laboratory Animals (National Research Council of the USA, 1996).

\section{Renal tissue staining}

The rat kidneys were fixed in $10 \%$ formalin and dehydrated with gradient ethanol. The tissue was embedded in paraffin and sectioned into $2-4 \mu \mathrm{m}$ slices. Then, the tissue slices were stained with periodic acid-Schiff. The mesangial hypercellularity index was used to assess the severity of hypercellular lesions as follows: 0 , no hypercellularity, with fewer than three cells per mesangial area; 1 , mild focal hypercellularity and $<50 \%$ of glomeruli with three to five cells per mesangial area; 2 , diffuse mild hypercellularity or prominent focal segmental hypercellularity with more than five cells per mesangial area; and 3, prominent, diffuse global hypercellularity. At least 20 glomeruli were selected for each section.

Immunohistochemical staining for RECA-1, PCNA and a-SMA was used to detect ECs proliferation. Paraffin sections $(2-4 \mu \mathrm{m})$ were dewaxed with xylene and ethanol, incubated with $3 \%$ hydrogen peroxide, and heated in a microwave for antigen repair. The sections were blocked with normal goat serum (ZLI9056, ORIGENE, CN) and incubated with RECA-1 antibody (1:100, Abcam, cat. ab22492), PCNA antibody (1:10000, Abcam, cat. ab92552) or a-SMA (1:10000, Abcam, cat. ab7817) overnight at $4{ }^{\circ} \mathrm{C}$. The indirect 
avidin-biotinylated peroxidase complex method (Vecta-Stain Elite ABC Kit, Vector Laboratories, Burlingame, CA, USA) with mouse anti-RECA-1 antibody, rabbit anti-PCNA antibody or mouse anti-a-SMA antibody (Santa Cruz Biotechnology, USA) was used to label RECA-1, PCNA or a-SMA. Phosphatebuffered saline (PBS) was used instead of primary antibodies as a negative control. The PCNA-positive rates are represented as the ratio of positive cells among all glomerular cells. ImageJ was used for RECA1 and a-SMA analysis. 20 glomeruli were counted in each section.

To assess the proliferation of ECs, RECA-1 and PCNA immunofluorescence co-staining was performed. Frozen sections were sequentially treated with $1 \%$ SDS and normal goat serum before being incubated with RECA-1 antibody (1:200, Abcam, cat. ab22492) and PCNA antibody (1:200, Abcam, cat. ab92552) overnight at $4{ }^{\circ} \mathrm{C}$. The sections were washed and probed with Cy3-conjugated mouse secondary antibody (red) and FITC-conjugated rabbit secondary antibody (green) at room temperature for $1 \mathrm{~h}$. DAPI was added to stain the nuclei. The tissue sections were imaged by confocal fluorescence microscopy. Each experiment was repeated three times.

\section{Cell culture}

Primary human renal mesangial cells (HRMCs) were purchased from ScienCell Research Laboratories (cat. 4200, USA) and cultured in mesangial cell medium (MCM, cat. 4201) supplemented with 10\% FBS (cat. 0010), $5 \mathrm{ml}$ of mesangial cell growth factor (MsCGS, cat. 4252) and $5 \mathrm{ml}$ of penicillin/streptomycin (P/S) solution (cat. 0503). Primary human glomerular endothelial cells (HRGECs) were purchased from ScienCell Research Laboratories (cat. 4000, USA) and cultured in endothelial cell medium (ECM, cat. 1001) supplemented with $10 \%$ FBS, $5 \mathrm{ml}$ of endothelial cell growth factor (ECGS, cat. 1052) and $5 \mathrm{ml}$ of $\mathrm{P} / \mathrm{S}$ solution. Cells were all maintained in $5 \% \mathrm{CO}_{2}$ at $37^{\circ} \mathrm{C}$. Transwells were purchased from Corning and used for coculturing. PDGF-BB (Peprotech, cat. 100-14b, $20 \mathrm{ng} / \mathrm{ml}$ ) and VEGFA (Peprotech, cat. 100 - 20, $50 \mathrm{ng} / \mathrm{ml}$ ) were used as irritants of HRMCs and HRGECs, respectively. Anti-VEGFA neutralizing antibody (R\&D, cat. ab-293-na, $3 \mu \mathrm{g} / \mathrm{ml}$ ) was added to the coculture system to bind to VEGFA. Recombinant human Angpt 1 (Prospec, cat. Cyt-074, $0.7 \mu \mathrm{g} / \mathrm{ml}$ ), which binds to Tie2 and competitively inhibits Angpt2, was used to inhibit ECs proliferation.

\section{Cell proliferation and migration analysis}

Cell proliferation was analyzed by the Click-iT® Plus EdU Alexa Fluor® 555 Imaging Kit (Invitrogen, cat. C10638) according to the manufacturer's instructions. The cells were cocultured in Transwell plates, and $10 \mu \mathrm{M}$ EdU was added to the medium for $24 \mathrm{~h}$. Then, the cells were fixed with $4 \%$ paraformaldehyde, permeabilized with $0.5 \%$ Triton X-100, and washed with $3 \%$ BSA. The cells were then incubated at room temperature in the Click-iT ${ }^{\circledR}$ reaction cocktail for $30 \mathrm{~min}$. DAPI was used to dye the nuclei. Cells were observed under a fluorescence microscope (Olympus, Tokyo, Japan). All experiments were repeated three times. To investigate cell migration, Transwells with a pore size of $8 \mu \mathrm{m}$ were used. After coculture, the filter membrane was stained with crystal violet dye. Cells above the filter membrane were removed, and cells on the bottom were observed and imaged under a microscope. All experiments were repeated three times. 


\section{VEGFA/VEGFR2, Angpt2/Tie2 and MAPK signaling pathway analysis}

Angpt2 (1:200, Abcam, cat. ab221154) immunofluorescence staining, as well as Tie2 (1:100, Biorbyt Ltd., orb247862) and p-tie2 (1:100, Biorbyt Ltd., orb7094) immunofluorescence costaining, were used to evaluate changes in Angpt2 expression and Tie2 phosphorylation after cell treatments. The gene expression levels were measured by real-time quantitative PCR (RT-qPCR). TRIzol reagent (Invitrogen) was used to lyse the cells and extract the RNA. The ReverTra Ace qPCR RT kit (Toyobo, Osaka, Japan) reverse transcribed the RNA into cDNA. RT-qPCR was performed using SYBR Select master mix (Life Technologies, California, USA) and an RT-qPCR detection system (ABI, Foster City, CA, USA). The primers information was constructed: VEGFA-human (5'-AGGGCAGAATCATCACGAAGT-3'; 5'AGGGTCTCGATTGGATGGCA-3'); Angpt2-human (5'-ACCCCACTGTTGCTAAGAAGA-3'; 5'CCATCCTCACGTCGCTGAATA-3'); VEGFA-rat(5'-GGGAGCAGAAAGCCCATGAA-3'; 5'GCTGGCTTTGGTGAGGTTTG-3'); PDGF-BB-rat(5'- ATGACCCGAGCACATTCTGG - 3'; 5'ACACCTCTGTACGCGTCTTG-3'). The $18 \mathrm{~s}$ gene was used as an internal control, and the expression of each target gene was calculated by the $2^{-\Delta \Delta C T}$ method. All experiments were repeated three times. Protein was measured by Western blotting. The glomeruli from renal tissue or cells were lysed with RIPA lysis buffer containing protease inhibitors and phosphatase inhibitors. After a 30 min incubation, the samples were centrifuged at $12000 \mathrm{r}$ and $4{ }^{\circ} \mathrm{C}$ for $30 \mathrm{~min}$. The protein concentration was determined by a BCA protein assay kit (Thermo Fisher Scientific, USA). Approximately $25 \mu \mathrm{g}$ of protein from each sample was separated by $8-15 \%$ SDS-PAGE. The samples were transferred from the SDS-PAGE gels to membranes. The membranes were blocked and incubated in antibodies against CD34(1:1000, Abcam, ab81289), Tie2 (1:1000, Biorbyt Ltd., orb247862), p-tie2 (1:1000, Biorbyt Ltd., orb7094), a-SMA (1:1000, Abcam, ab7817), VEGFR2 (1:1000, CST, 2479s) p-VEGFR2 (1:1000, CST, 2478s), MAPK (1:1000, CST, 9102s), p-MAPK $(1: 1000, C S T, 9101 s)$ and $\beta$-actin overnight at $4{ }^{\circ} \mathrm{C}$. Finally, the membranes were incubated with secondary antibody at room temperature for $1 \mathrm{~h}$. ImageJ was used for blot analysis. All experiments were repeated three times.

\section{Statistical analysis}

All data analyses were performed using GraphPad (version 5.0; GraphPad, San Diego, CA). The data are expressed as the means $\pm S D$. Comparisons among the groups were conducted using one-way ANOVA. A value of $P<0.05$ was considered significant.

\section{Abbreviations}




\section{Declarations}

\begin{tabular}{|ll|}
\hline MCs & Mesangial cells \\
\hline ECs & Endothelial cells \\
\hline ESRD & End-stage renal disease \\
VEGF & Vascular endothelial growth factor \\
\hline Angpt & Angiopoietin \\
\hline HRMC & Human renal mesangial cell \\
\hline HRGEC & Human renal glomerular endothelial cell \\
\hline CKD & Chronic kidney disease \\
\hline TMA & Thrombotic microangiopathy \\
\hline GBM & Glomerular basement membrane \\
\hline AKI & Acute kidney injury \\
\hline
\end{tabular}

\section{Ethics approval and consent to participate}

The experiments were performed according to the Guide for the Care and Use of Laboratory Animals (National Research Council of the USA, 1996).

\section{Consent for publication}

Not applicable.

\section{Availability of data and metarials}

All data generated or analysed during this study are included in this published article and its supplementary information files.

\section{Competing interests}

The authors declare that they have no competing interests.

\section{Funding}

This work was supported by the National Natural Science Foundation of China (No. 81330019ه 81830019), the Science and Technology Project of Beijing, China (D18110700010000, D181100000118002, D181100000118004).

\section{Authors' contribution}


Yinghua Zhao, Lingling Wu and Xiangmei Chen designed research; Yinghua Zhao, Bo Fu and Pu Chen analyzed data and performed research; Yinghua Zhao and Lingling Wu wrote the paper; Qinggang Li, Qing Ouyang, Chuyue Zhang, Guangyan Cai, and Xiangmei Chen optimized the paper.

\section{Acknowledgement}

Not applicable.

\section{Disclosure}

Not applicable.

\section{References}

1. Bai J, et al., Effect of Huaier On the Proliferation of Mesangial Cells in Anti-Thy-1 Nephritis. Cellular physiology and biochemistry: international journal of experimental cellular physiology, biochemistry, and pharmacology, 2017. 42(6): p. 2441-2452.

2. Guo L, et al. Targeted delivery of celastrol to mesangial cells is effective against mesangioproliferative glomerulonephritis. Nature communications. 2017;8(1):878.

3. Qin $D$, et al. Aldosterone mediates glomerular inflammation in experimental mesangial proliferative glomerulonephritis. J Nephrol. 2013;26(1):199-206.

4. Jin $\mathrm{M}$, et al. Metanephric mesenchyme-derived Foxd1 mesangial precursor cells alleviate mesangial proliferative glomerulonephritis. J Mol Med. 2019;97(4):553-61.

5. Lu Y, et al. The role of transcriptional factor D-site-binding protein in circadian CCL2 gene expression in anti-Thy1 nephritis. Cell Mol Immunol. 2019;16(9):735-45.

6. Carmeliet P, Collen D. Vascular development and disorders: molecular analysis and pathogenic insights. Kidney international. 1998;53(6):1519-49.

7. Verma SK, Molitoris BA. Renal endothelial injury and microvascular dysfunction in acute kidney injury. Seminars in nephrology, 2015. 35(1).

8. Endemann DH, Schiffrin EL. Endothelial dysfunction. Journal of the American Society of Nephrology: JASN. 2004;15(8):1983-92.

9. Daniel C, et al. Thrombospondin-2 therapy ameliorates experimental glomerulonephritis via inhibition of cell proliferation, inflammation, and TGF-beta activation. Am J Physiol Renal Physiol. 2009;297(5):F1299-309.

10. Mii A, et al. Angiotensin II receptor blockade inhibits acute glomerular injuries with the alteration of receptor expression. Lab Invest. 2009;89(2):164-77.

11. Iruela-Arispe $L$, et al. Participation of glomerular endothelial cells in the capillary repair of glomerulonephritis. Am J Pathol. 1995;147(6):1715-27.

12. Wada $Y$, et al. Impairment of vascular regeneration precedes progressive glomerulosclerosis in antiThy 1 glomerulonephritis. Kidney international. 2002;61(2):432-43. 
13. Yao J, Oite T, Kitamura M. Gap junctional intercellular communication in the juxtaglomerular apparatus. Am J Physiol Renal Physiol. 2009;296(5):F939-46.

14. Majumder S, Advani A. VEGF and the diabetic kidney: More than too much of a good thing. $J$ Diabetes Complicat. 2017;31(1):273-9.

15. Veron D, Tufro A. Reply to letter to editor comment on «Cellular and molecular aspects of diabetic nephropathy; the role of VEGF-A». Nefrologia: publicacion oficial de la Sociedad Espanola Nefrologia, 2016. 36(6): pp. 706-7.

16. Eklund L, Kangas J, Saharinen P, Angiopoietin-Tie signalling in the cardiovascular and lymphatic systems. Clinical science (London, England: 1979), 2017. 131(1).

17. Augustin HG, et al. Control of vascular morphogenesis and homeostasis through the angiopoietin-Tie system. Nat Rev Mol Cell Biol. 2009;10(3):165-77.

18. Ghosh CC, et al. Impaired function of the Tie-2 receptor contributes to vascular leakage and lethality in anthrax. Proc Natl Acad Sci USA. 2012;109(25):10024-9.

19. Felcht $M$, et al. Angiopoietin-2 differentially regulates angiogenesis through TIE2 and integrin signaling. J Clin Investig. 2012;122(6):1991-2005.

20. Tsai Y-C, et al., Angiopoietin-2, Renal Deterioration, Major Adverse Cardiovascular Events and AllCause Mortality in Patients with Diabetic Nephropathy. Kidney \& blood pressure research, 2018. 43(2): p. 545-554.

21. Khan KA, Kerbel RS. Improving immunotherapy outcomes with anti-angiogenic treatments and vice versa. Nat Rev Clin Oncol. 2018;15(5):310-24.

22. Tsai Y-C, et al. Angpt2 Induces Mesangial Cell Apoptosis through the MicroRNA-33-5p-SOCS5 Loop in Diabetic Nephropathy. Molecular therapy. Nucleic acids. 2018;13:543-55.

23. Prommer $\mathrm{H}-\mathrm{U}$, et al. Chronic kidney disease induces a systemic microangiopathy, tissue hypoxia and dysfunctional angiogenesis. Scientific reports. 2018;8(1):5317.

24. Alfieri A, et al. Angiopoietin-1 regulates microvascular reactivity and protects the microcirculation during acute endothelial dysfunction: role of eNOS and VE-cadherin. Pharmacological research. 2014;80:43-51.

25. Dekker NAM, et al. Vasculotide, an angiopoietin-1 mimetic, reduces pulmonary vascular leakage and preserves microcirculatory perfusion during cardiopulmonary bypass in rats. $\mathrm{Br} \mathrm{J}$ Anaesth. 2018;121(5):1041-51.

26. Syrjälä SO, et al., Donor Heart Treatment With COMP-Ang1 Limits Ischemia-Reperfusion Injury and Rejection of Cardiac Allografts. American journal of transplantation: official journal of the American Society of Transplantation and the American Society of Transplant Surgeons, 2015. 15(8): p. 20752084.

27. Moon HE, et al. COMP-Ang1 Potentiates EPC Treatment of Ischemic Brain Injury by Enhancing Angiogenesis Through Activating AKT-mTOR Pathway and Promoting Vascular Migration Through Activating Tie2-FAK Pathway. Experimental neurobiology. 2015;24(1):55-70. 
28. Trieu M, et al. Vasculotide, an Angiopoietin-1 Mimetic, Restores Microcirculatory Perfusion and Microvascular Leakage and Decreases Fluid Resuscitation Requirements in Hemorrhagic Shock. Anesthesiology. 2018;128(2):361-74.

29. Rübig E, et al., The Synthetic Tie2 Agonist Peptide Vasculotide Protects Renal Vascular Barrier Function In Experimental Acute Kidney Injury. Scientific reports, 2016. 6: p. 22111.

30. Tsai Y-C, et al. Angiopoietin-2, Angiopoietin-1 and subclinical cardiovascular disease in Chronic Kidney Disease. Scientific reports. 2016;6:39400.

31. Lin C-Y, et al. ADAM9 promotes lung cancer progression through vascular remodeling by VEGFA, ANGPT2, and PLAT. Scientific reports. 2017;7(1):15108.

32. Schmittnaegel $\mathrm{M}$, et al., Dual angiopoietin-2 and VEGFA inhibition elicits antitumor immunity that is enhanced by PD-1 checkpoint blockade. Science translational medicine, 2017. 9(385).

33. Hugo $\mathrm{CP}$, et al. Thrombospondin peptides are potent inhibitors of mesangial and glomerular endothelial cell proliferation in vitro and in vivo. Kidney international. 1999;55(6):2236-49.

34. Eremina V, et al. Glomerular-specific alterations of VEGF-A expression lead to distinct congenital and acquired renal diseases. J Clin Investig. 2003;111(5):707-16.

35. Ferrara N. Role of vascular endothelial growth factor in regulation of physiological angiogenesis. American journal of physiology Cell physiology. 2001;280(6):C1358-66.

36. Wang $L$, et al. Podocytes protect glomerular endothelial cells from hypoxic injury via deSUMOylation of HIF-1a signaling. Int J Biochem Cell Biol. 2015;58:17-27.

37. Liu E, et al. Increased expression of vascular endothelial growth factor in kidney leads to progressive impairment of glomerular functions. Journal of the American Society of Nephrology: JASN. 2007;18(7):2094-104.

38. Carranza K, et al. Cellular and molecular aspects of diabetic nephropathy; the role of VEGF-A. Nefrologia: publicacion oficial de la Sociedad Espanola Nefrologia. 2015;35(2):131-8.

39. Torres-Salido MT, et al., Urinary Neuropilin-1: A Predictive Biomarker for Renal Outcome in Lupus Nephritis. International journal of molecular sciences, 2019. 20(18).

40. Fiedler U, et al. Angiopoietin-2 sensitizes endothelial cells to TNF-alpha and has a crucial role in the induction of inflammation. Nature medicine. 2006;12(2):235-9.

41. Kümpers $P$, et al. The Tie2 receptor antagonist angiopoietin 2 facilitates vascular inflammation in systemic lupus erythematosus. Ann Rheum Dis. 2009;68(10):1638-43.

42. Lovric $S$, et al. Removal of elevated circulating angiopoietin-2 by plasma exchange-a pilot study in critically ill patients with thrombotic microangiopathy and anti-glomerular basement membrane disease. Thromb Haemost. 2010;104(5):1038-43.

43. El-Banawy HS, et al. Angiopoietin-2, endothelial dysfunction and renal involvement in patients with systemic lupus erythematosus. J Nephrol. 2012;25(4):541-50.

44. David S, et al., Angiopoietin-2 levels predict mortality in CKD patients. Nephrology, dialysis, transplantation: official publication of the European Dialysis and Transplant Association - European 
Renal Association, 2012. 27(5): p. 1867-1872.

45. Carmeliet P, Jain RK. Molecular mechanisms and clinical applications of angiogenesis. Nature. 2011;473(7347):298-307.

46. Chae S-S, et al. Angiopoietin-2 interferes with anti-VEGFR2-induced vessel normalization and survival benefit in mice bearing gliomas. Clinical cancer research: an official journal of the American Association for Cancer Research. 2010;16(14):3618-27.

47. Kienast $Y$, et al. Ang-2-VEGF-A CrossMab, a novel bispecific human IgG1 antibody blocking VEGF-A and Ang-2 functions simultaneously, mediates potent antitumor, antiangiogenic, and antimetastatic efficacy. Clinical cancer research: an official journal of the American Association for Cancer Research. 2013;19(24):6730-40.

48. Jeansson $M$, et al. Angiopoietin- 1 is essential in mouse vasculature during development and in response to injury. J Clin Investig. 2011;121(6):2278-89.

49. Robinson-Cohen C, et al. Association of markers of endothelial dysregulation Ang1 and Ang2 with acute kidney injury in critically ill patients. Crit Care (London England). 2016;20(1):207.

50. Chiang W-C, et al., Angiopoietin 1 influences ischemic reperfusion renal injury via modulating endothelium survival and regeneration. Molecular medicine (Cambridge, Mass.), 2019. 25(1): p. 5.

51. Lee $\mathrm{S}$, et al., Renoprotective effect of COMP-angiopoietin-1 in $\mathrm{db} / \mathrm{db}$ mice with type 2 diabetes. Nephrology, dialysis, transplantation: official publication of the European Dialysis and Transplant Association - European Renal Association, 2007. 22(2): p. 396-408.

52. Zhang L, Nakazawa K, Shigematsu H. Participation of endothelial cells and transformed mesangial cells in remodeling of glomerular capillary loops in Thy- 1 nephritis. Pathology international. 2001;51(4):227-39.

\section{Figures}




\section{Figure 1}

a

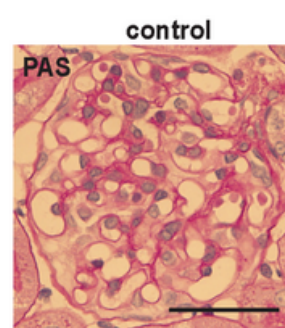

RECA-1

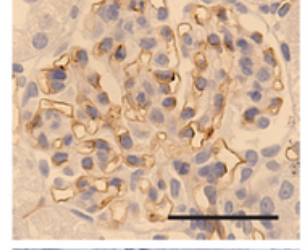

PCNA

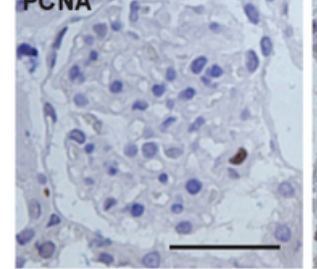

b

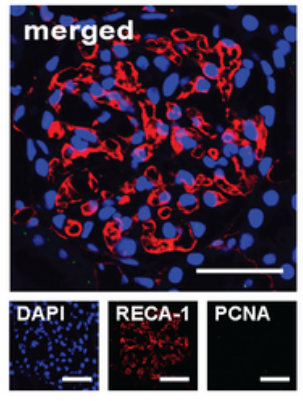

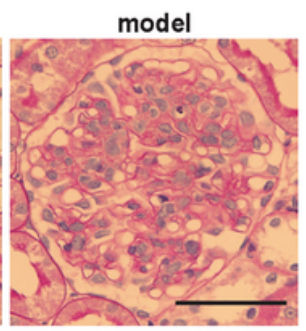
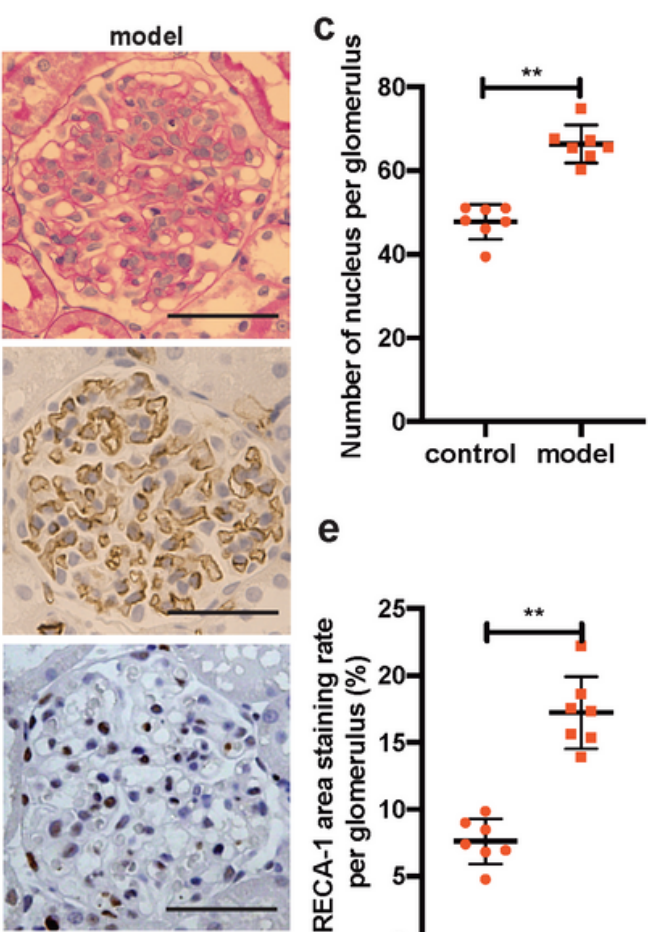

e

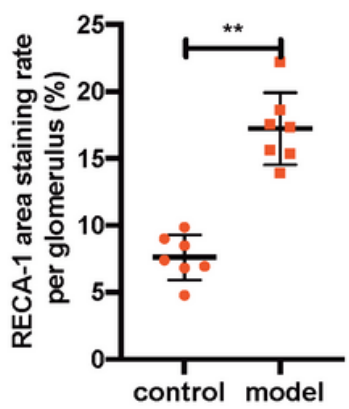

f

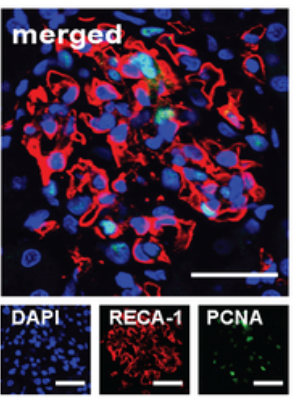

d
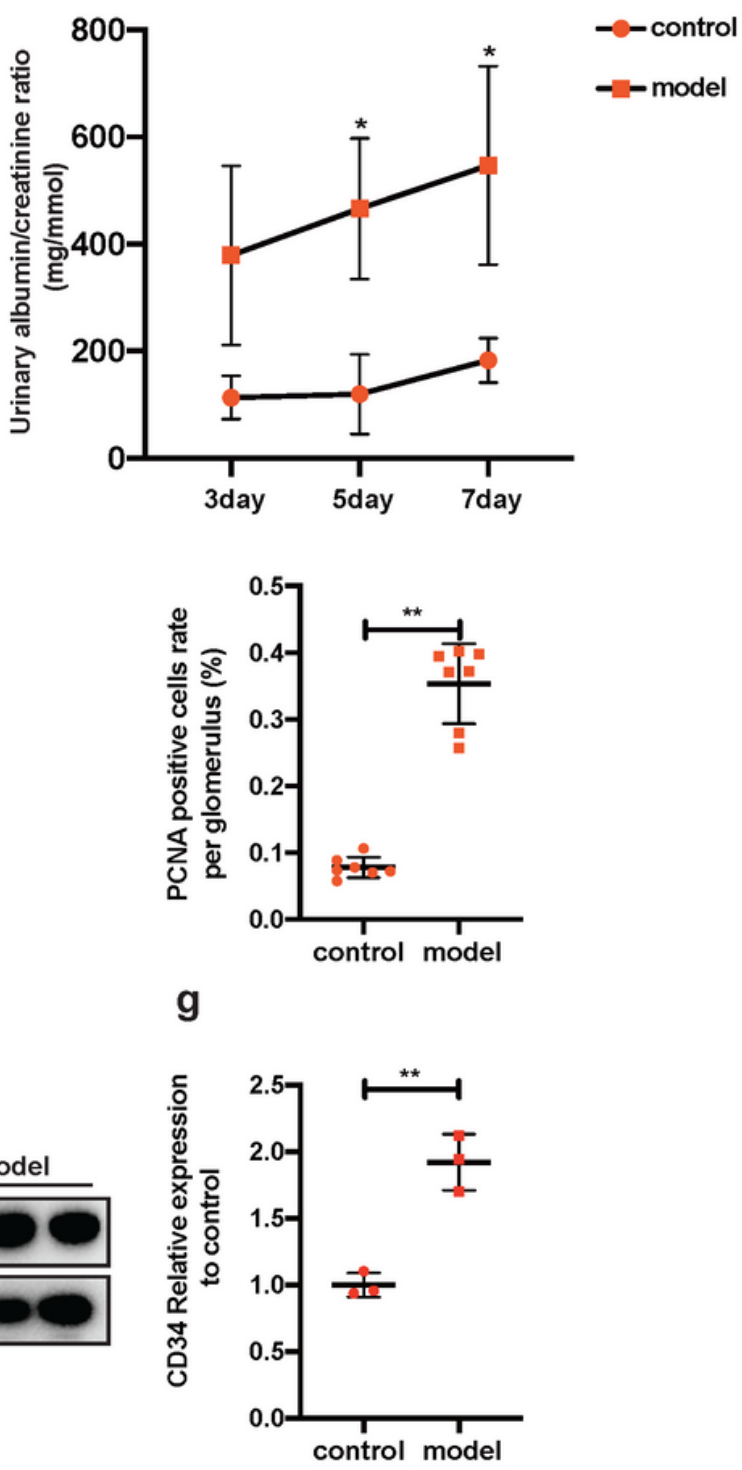

\section{Figure 1}

g

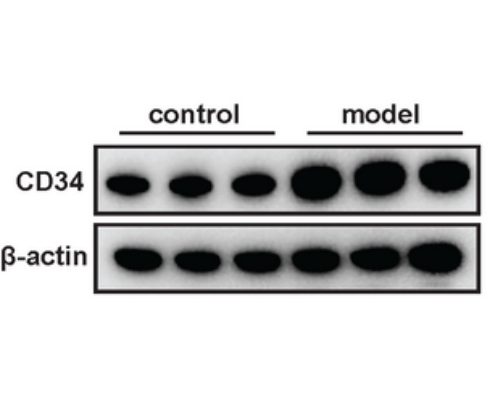


the mean values $( \pm S D)$, ${ }^{*} P<0.5$, control vs model. ( $f$ and $g$ ) Western blot results showed that CD34 expression was significantly increased in the model group. The results are presented as the mean values $( \pm S D),{ }^{\star} \times P<0.01$, control vs model.

\section{Figure 2}

a
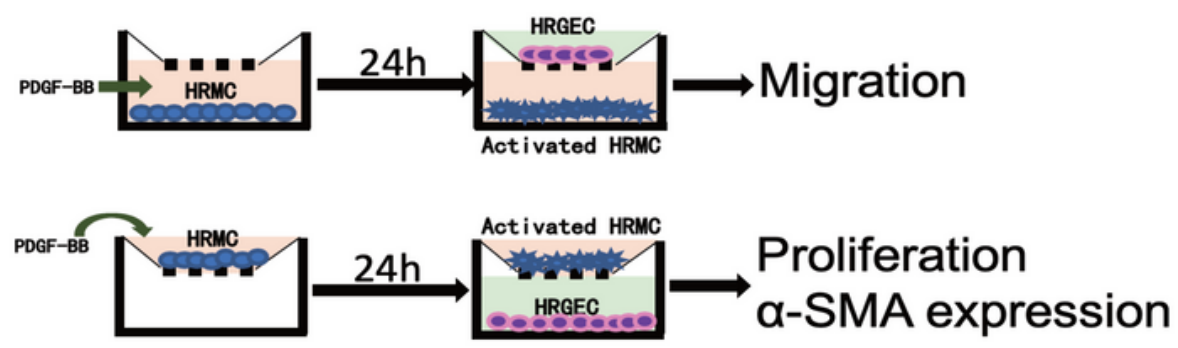

b

d

e
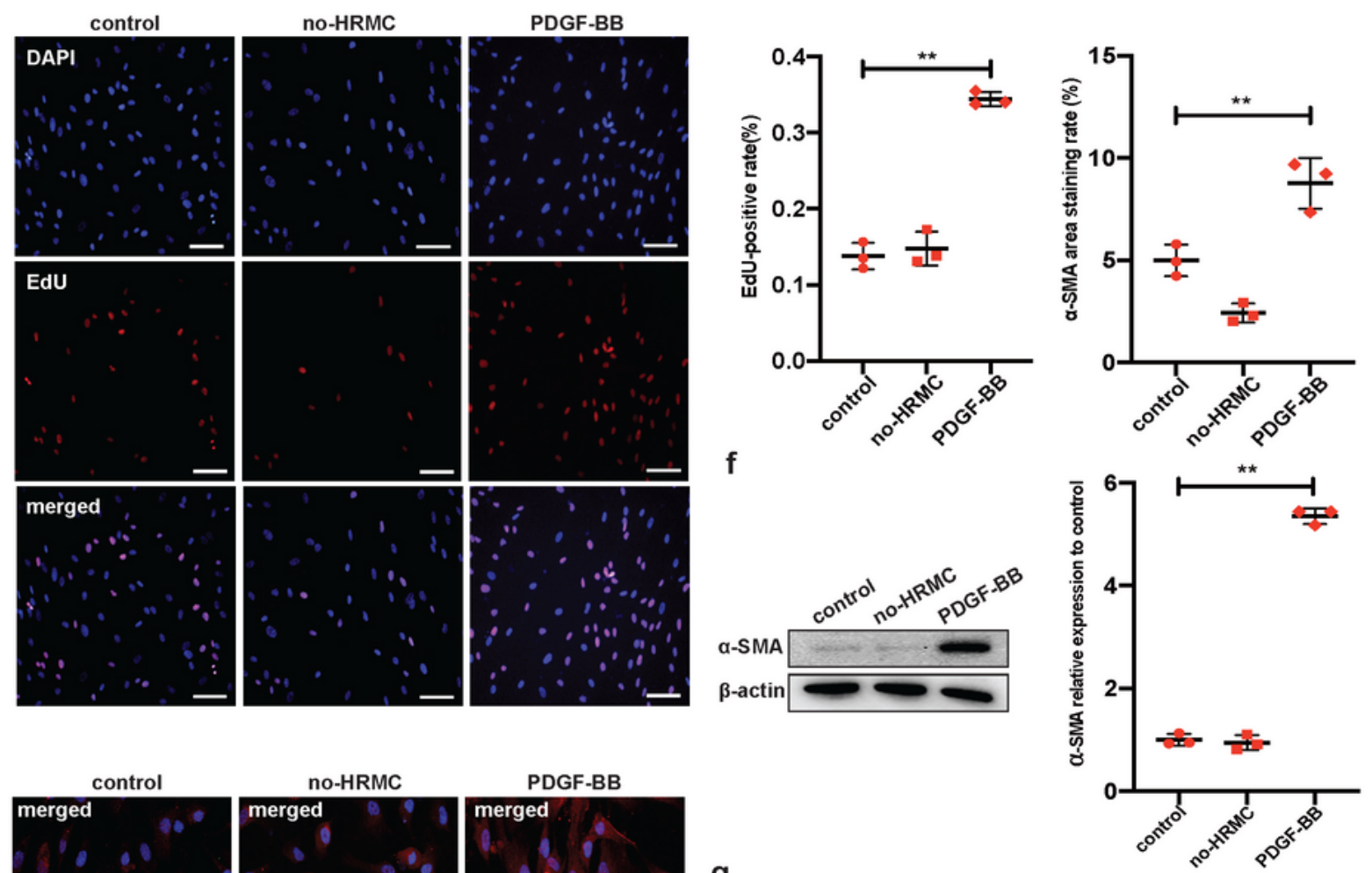

c
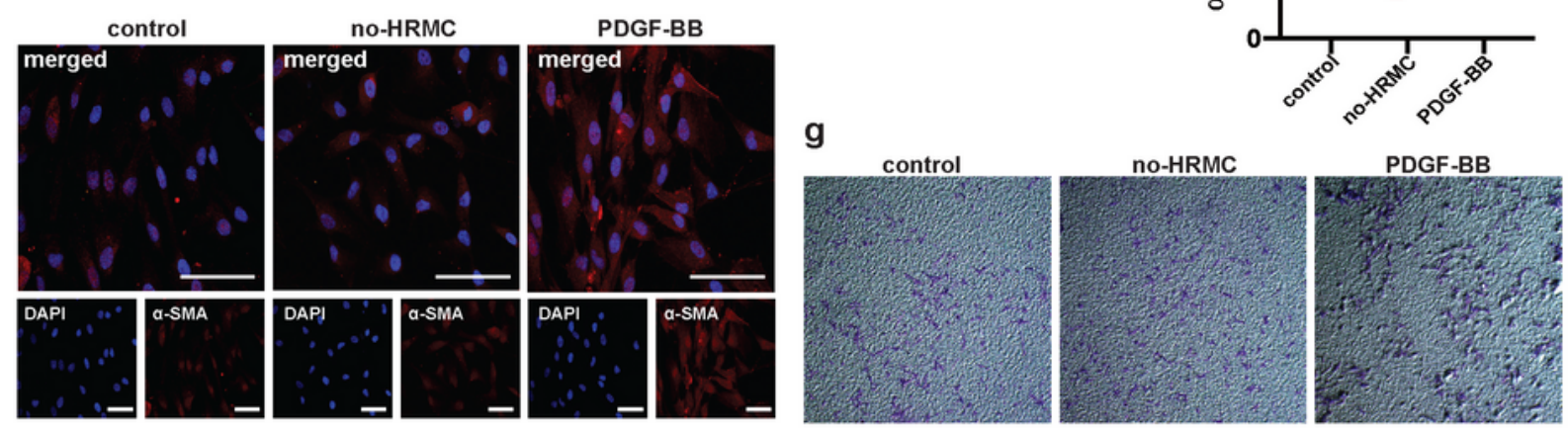

Figure 2

Activated MCs promote the ECs migration, proliferation and a-SMA expression. (a) The lower chamber with activated MCs was cocultured with the upper compartment with ECs to investigate the influence of 
MCs on ECs migration. The upper compartment with activated MCs was cocultured with the lower chamber with ECs to investigate the influence of MCs on ECs proliferation and a-SMA expression. (b)\&(d) EdU incorporation assay of ECs (red EdU, blue DAPI, pink merged). After being cocultured with activated MCs, ECs proliferation was greatly enhanced. The results are presented as the mean values ( $\pm S D$ ), $\star * P<0.01$, control vs PDGF-BB. $\times 200$ original magnification. (c, e and f) Immunofluorescence and Western blot analysis of a-SMA expression levels in ECs. After being cocultured with activated MCs, a-SMA expression in ECs was increased. The results are presented as the mean values ( $\pm S D),{ }^{*} P<0.01$, control vs PDGF-BB. $\times 400$ original magnification. (g) Transwell migration assay of ECs (cells passing through membranes were dyed by crystal violet). After being cocultured with activated MCs, the number of ECs passing through membranes was increased. Control, ECs were cocultured with resting MCs; no-HRMC, ECs were cultured separately; PDGF-BB, ECs were cocultured with PDGF-BB-activated MCs. 


\section{Figure 3}

a
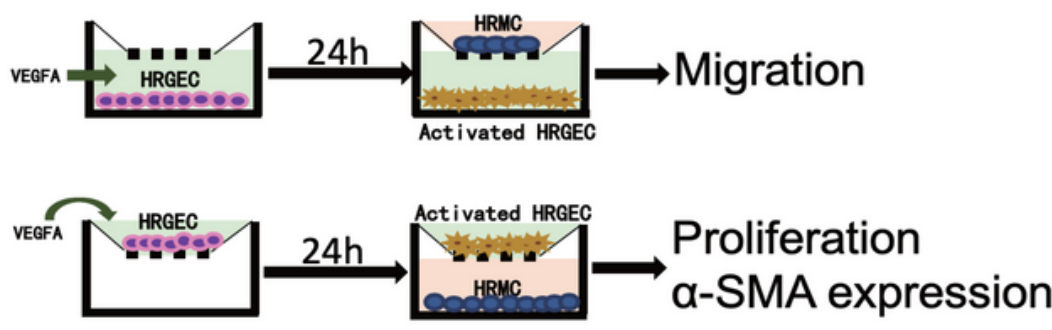

b
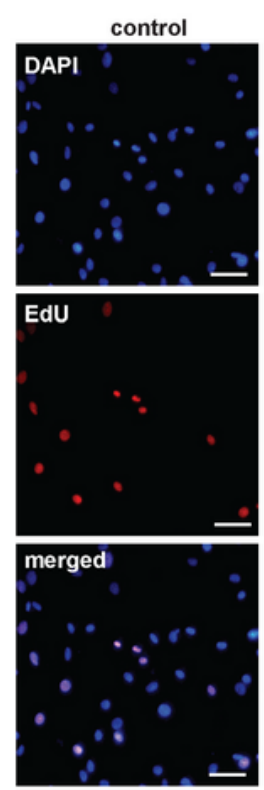

C
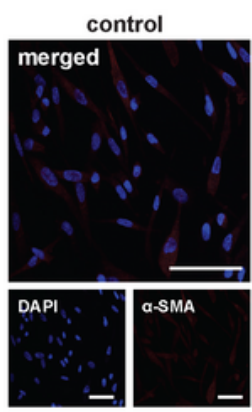
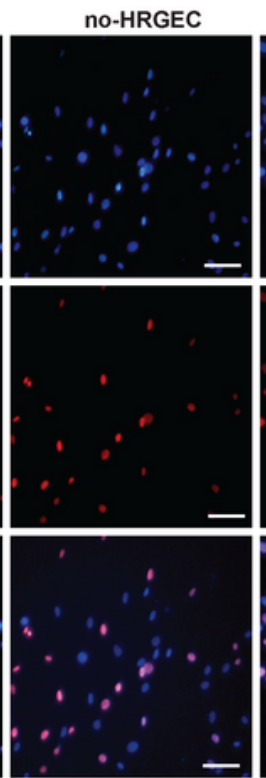

no-HRGEC
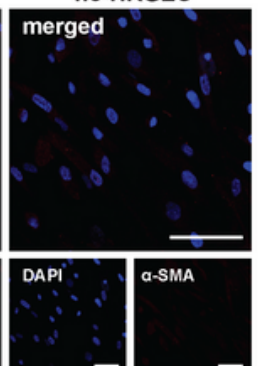

d

VEGFA
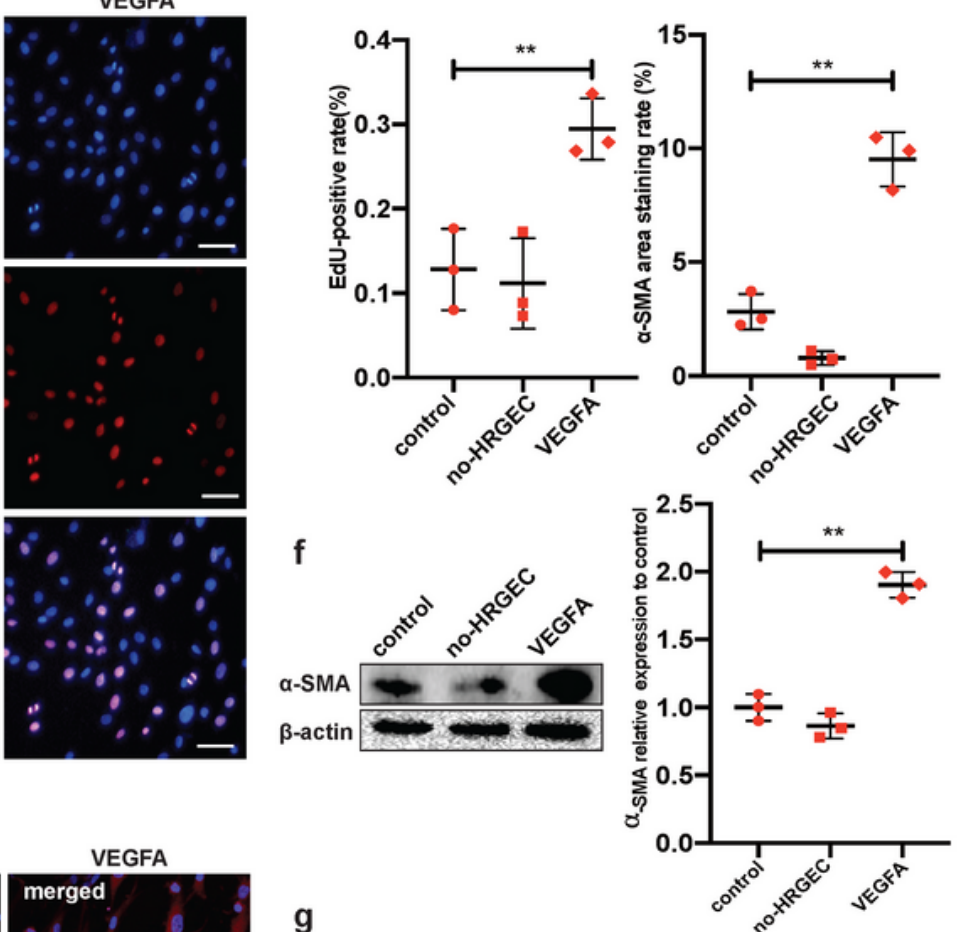

g
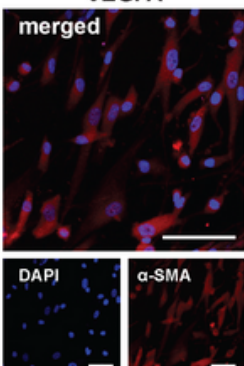

e

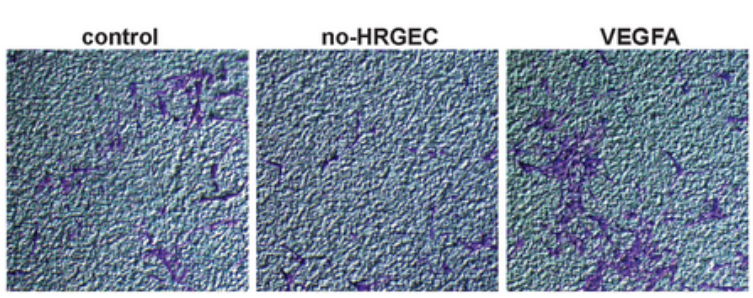

\section{Figure 3}

Activated ECs promote the MCs migration, proliferation and a-SMA expression. (a) The lower chamber with activated ECs was cocultured with the upper compartment with MCs to investigate the influence of ECs on MCs migration. The upper compartment with activated ECs was cocultured with the lower chamber with MCs to investigate the influence of ECs on MCs proliferation and a-SMA expression. (b and d) EdU incorporation assay of MCs (red EdU, blue DAPI, pink merged). After being cocultured with 
activated ECs, MCs proliferation was greatly enhanced. The results are presented as the mean values $( \pm S D), * * P<0.01$, control vs VEGFA. $\times 200$ original magnification. (c, e and f) Immunofluorescence and Western blot analysis of a-SMA expression levels in MCs. After being cocultured with activated ECs, the aSMA expression of MCs was increased. The results are presented as the mean values $( \pm S D),{ }^{*} P<0.01$, control vs VEGFA. $\times 400$ original magnification. (g) Transwell migration assay of MCs (cells passing through membranes were dyed by crystal violet). After being cocultured with activated ECs, the number of MCs passing through membranes was increased. Control, MCs were cocultured with resting ECs; noHRGEC, MCs were cultured separately; VEGFA, MCs were cocultured with VEGFA-activated ECs. 


\section{Figure 4}

a

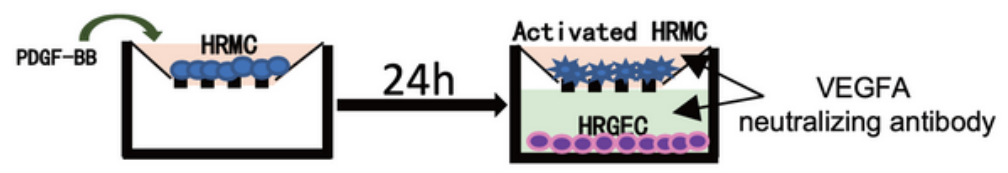

b

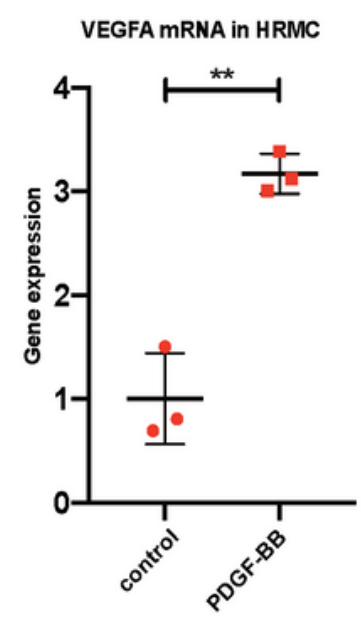

d

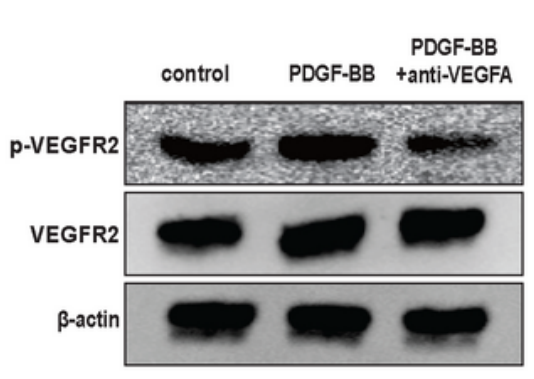

c
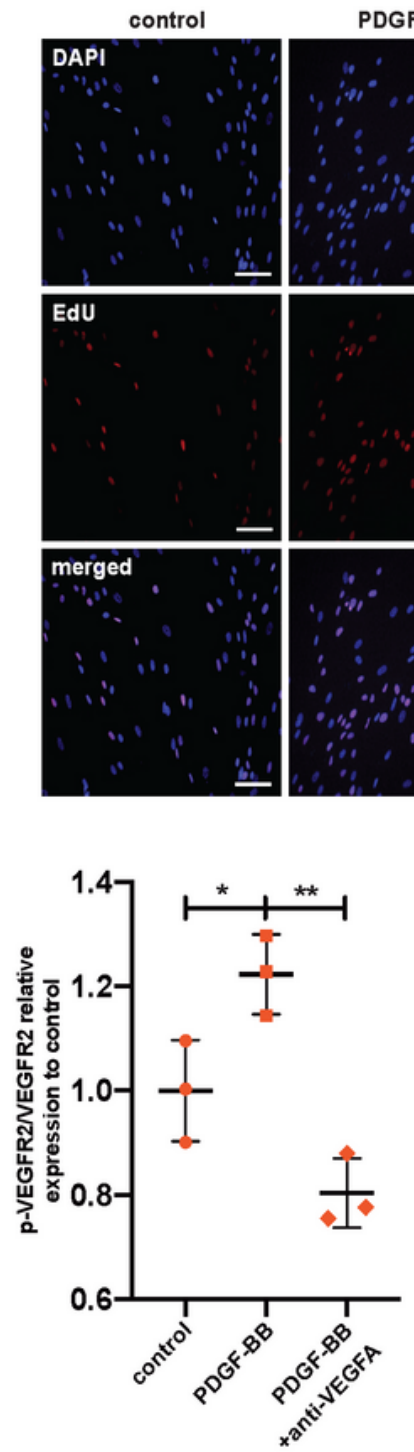

PDGF-BB

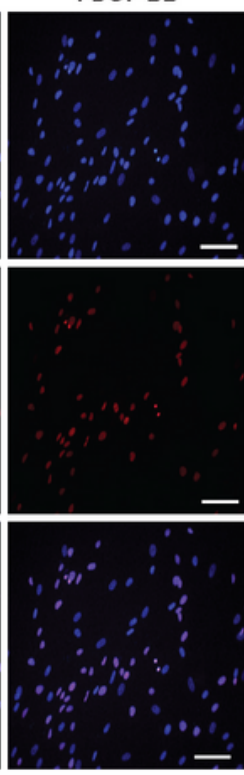

e
PDGF-BB
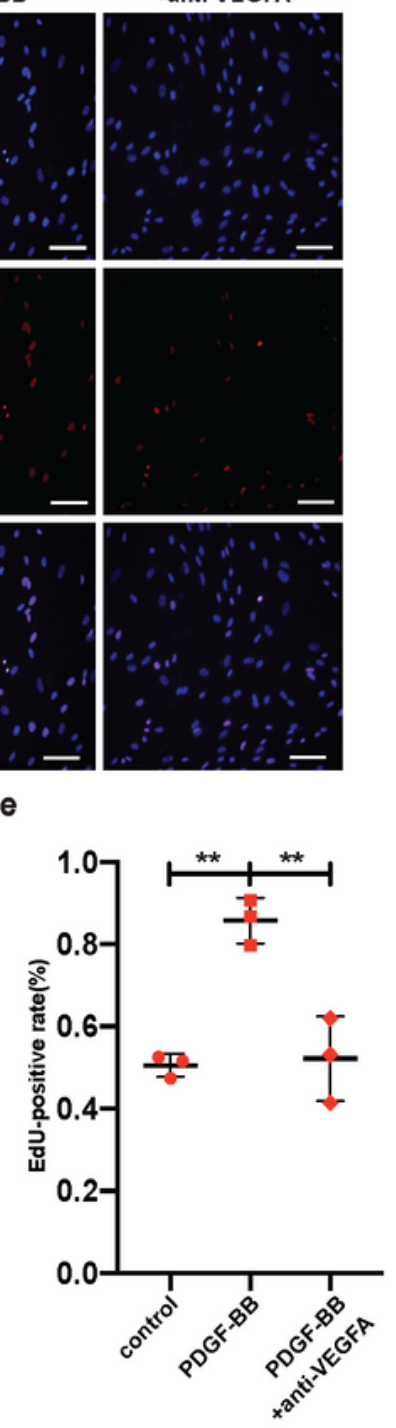

\section{Figure 4}

VEGFA/VEGFR2 signaling is involved in the crosstalk between MCs and ECs. (a) The upper compartment with activated MCs was cocultured with the lower chamber with ECs, and VEGFA neutralizing antibody was added in the cocultured system. (b) The expression levels of VEGFA mRNA in activated MCs significantly increased. The results are presented as the mean values $( \pm S D),{ }^{\star}{ }^{*} P<0.01$, control vs PDGFBB. Control, resting MCs; PDGF-BB, MCs was activated by PDGF-BB. (c and e) EdU incorporation assay of 
ECs (red EdU, blue DAPI, pink merged). The proliferation of ECs was greatly decreased after the VEGFA neutralizing antibodies were added to the coculture system. The results are presented as the mean values $( \pm S D), * * P<0.01$, control vs PDGF-BB, PDGF-BB vs PDGF-BB+anti-VEGFA. $\times 200$ original magnification. (d) Western blot results showed that $p$-VEGFR2 expression was significantly increased when ECs cocultured with activated MCs, while p-VEGFR2 expression decreased after adding VEGFA neutralizing antibody. The results are presented as the mean values $( \pm S D),{ }^{*} P<0.5$, control vs PDGF-BB, ${ }^{*} P<0.01$, PDGF-BB vs PDGFBB+anti-VEGFA. Control, ECs was cocultured with resting MCs; PDGF-BB, ECs was cocultured with PDGFBB-activated MCs; PDGF-BB+anti-VEGFA, VEGFA neutralizing antibody was added in the cocultured system.

\section{Figure 5}

a

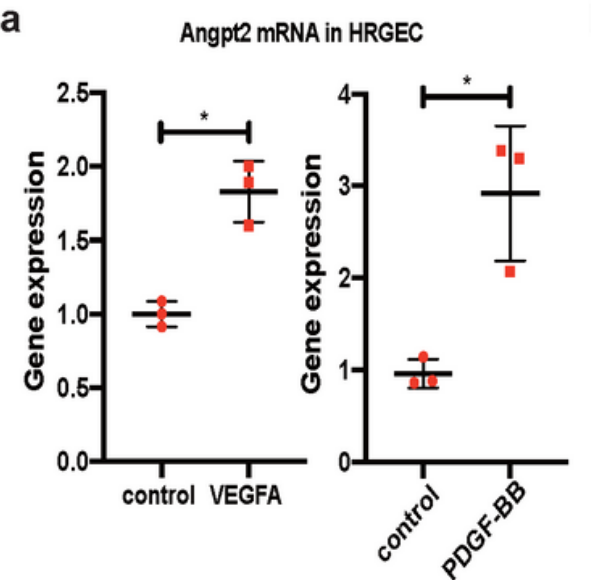

C

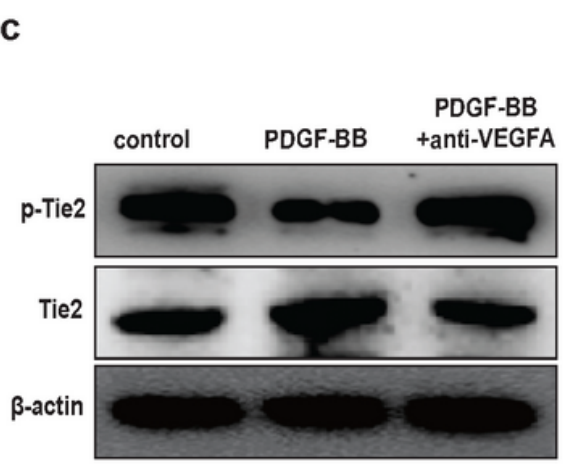

b
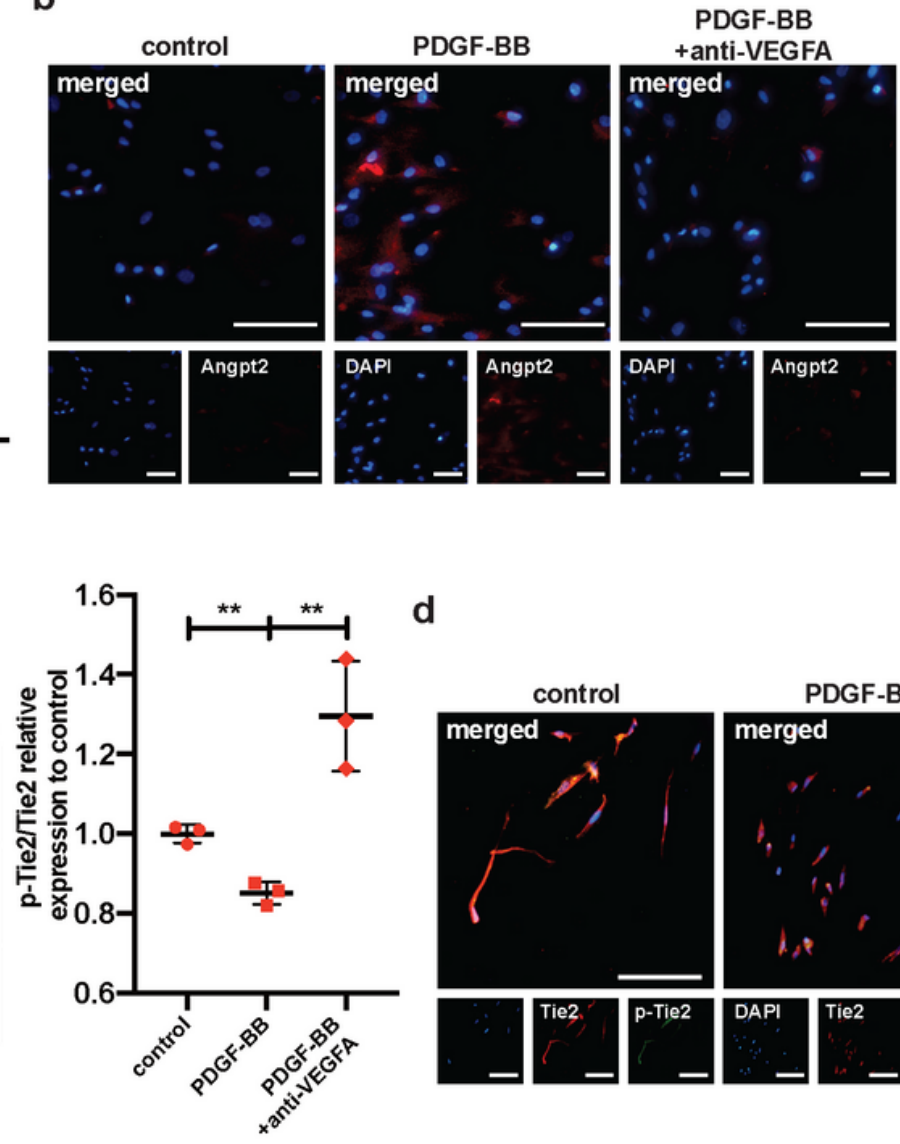

d

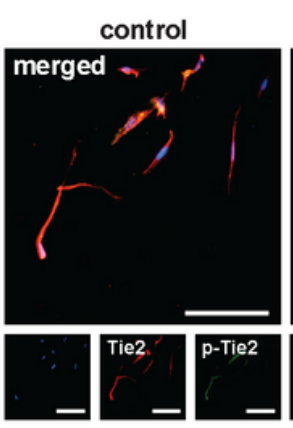

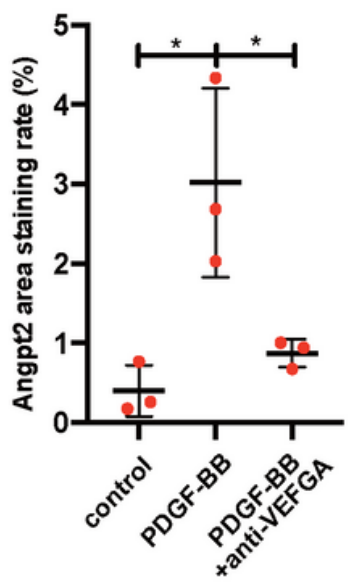
+ anti-VEGFA
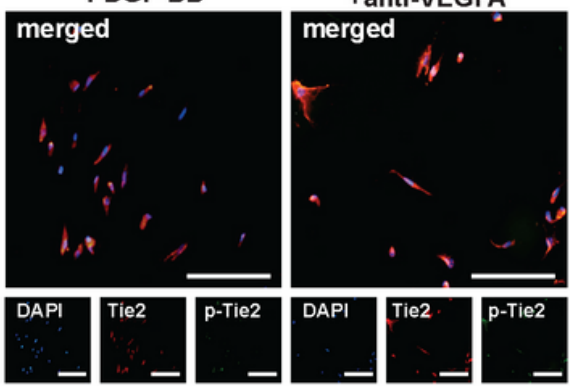

\section{Figure 5}

Angpt2/Tie2 signaling is involved in the crosstalk between MCs and ECs. (a) The expression of Angpt2 mRNA in ECs can be promoted by VEGFA. The results are presented as the mean values $( \pm S D),{ }^{*} P<0.5$, 
control vs VEGFA. Control, resting ECs; VEGFA, ECs was activated by VEGFA. The expression of Angpt2 mRNA in ECs can be promoted by cocultured with activated MCs. The results are presented as the mean values $( \pm S D)$, ${ }^{*}<<0.5$, control vs PDGF-BB. Control, ECs was cocultured with resting MCs; PDGF-BB, ECs was cocultured with PDGF-BB-activated MCs. (b and $d$ ) Immunofluorescence staining results showed that the PDGF-BB-activated MCs increased the expression of Angpt2 in ECs but lowered the phosphorylation levels of Tie2 on ECs. The VEGFA neutralizing antibody can significantly inhibit the effect of activated MCs on ECs. The results are presented as the mean values ( $\pm S D)$, ${ }^{*} P<0.5$, control vs PDGF-BB, PDGF-BB vs PDGF-BB+anti-VEGFA. $\times 400$ original magnification. (c) Western blot showed that the phosphorylation levels of Tie2 on ECs were increased when VEGFA neutralizing antibody was added. The results are presented as the mean values $( \pm S D),{ }^{* *} \mathrm{P}<0.01$, control vs PDGF-BB, PDGF-BB vs PDGF-BB+anti-VEGFA. Control, ECs was cocultured with resting MCs; PDGF-BB, ECs was cocultured with PDGF-BB-activated MCs; PDGF-BB+anti-VEGFA, VEGFA neutralizing antibody was added in the cocultured system. 


\section{Figure 6}

a
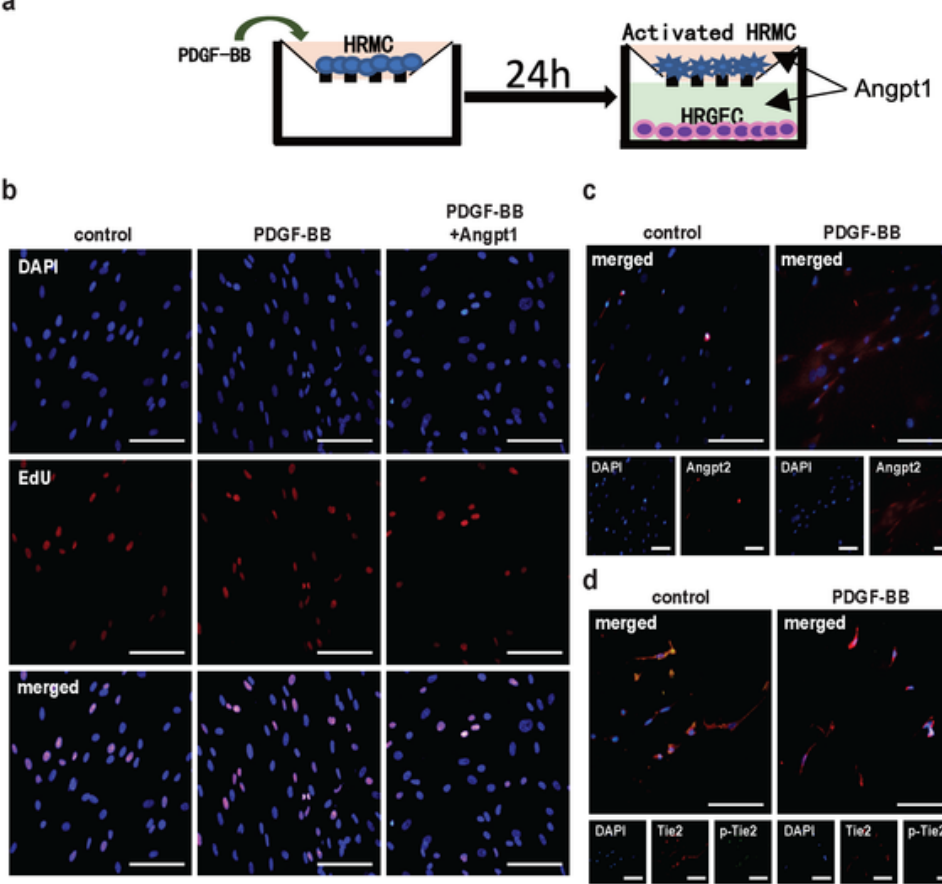

C
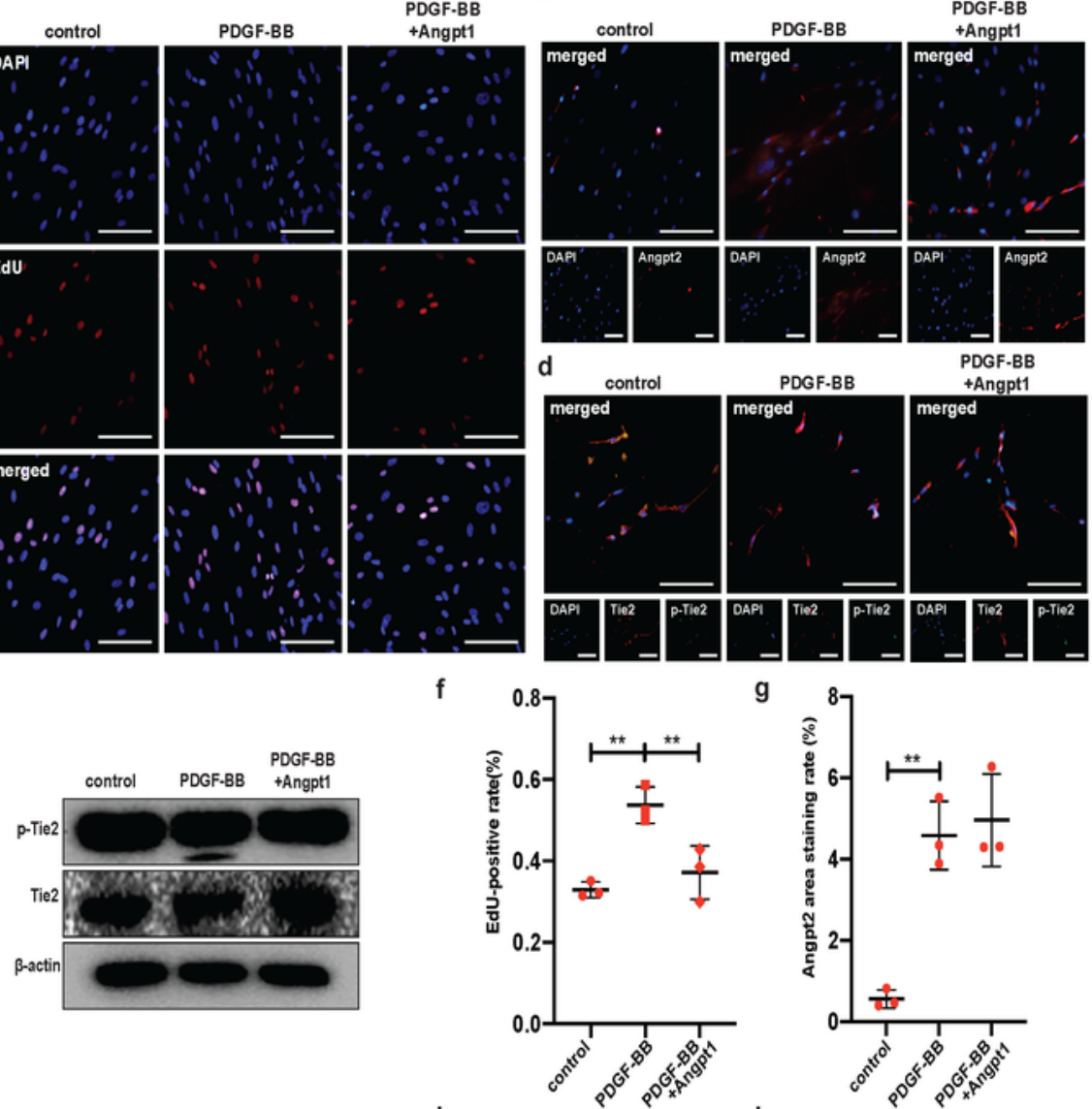

h
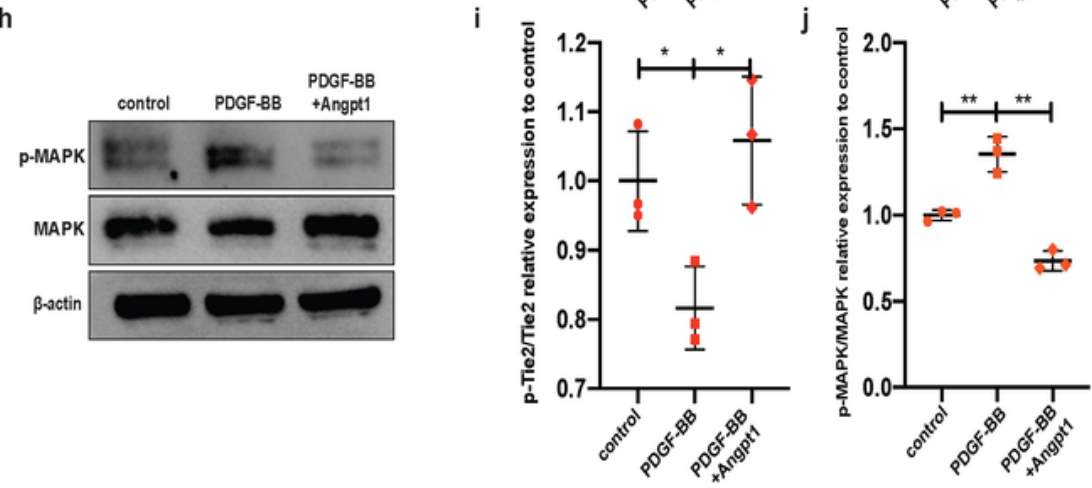

\section{Figure 6}

Angpt1 can effectively alleviate the proliferation-promoting effect of activated MCs on ECs. (a) The upper compartment with activated MCs was cocultured with the lower chamber with ECs, and Angpt1 was added to the coculture system. (b and f) EdU incorporation assay of ECs (red EdU, blue DAPI, pink merged). The proliferation of ECs was definitely inhibited after Angpt1 was added in the co-culture system. The results are presented as the mean values $( \pm S D), * * P<0.01$, control vs PDGF-BB, PDGF-BB vs 
PDGF-BB+Angpt1. (c, d and g) Immunofluorescence results showed that after the addition of Angpt1, the expression of Angpt2 in ECs was not changed, but the phosphorylation level of Tie2 was significantly increased. $\times 400$ original magnification. (e and i) Western blot results showed that after the addition of Angpt1, the phosphorylation level of Tie2 was significantly increased. The results are presented as the mean values $( \pm S D),{ }^{*} P<0.01$, control vs PDGF-BB, PDGF-BB vs PDGF-BB+Angpt1. (h and j) Western blot results showed that after the addition of Angpt1, the phosphorylation level of MAPK was significantly decreased. The results are presented as the mean values $( \pm S D),{ }^{*} P<0.5$, control vs PDGF-BB, PDGF-BB vs PDGF-BB+Angpt1. Control, ECs was cocultured with resting MCs; PDGF-BB, ECs was cocultured with PDGF-BB-activated MCs; PDGF-BB+Angpt1, Angpt1 was added in the cocultured system. 
a

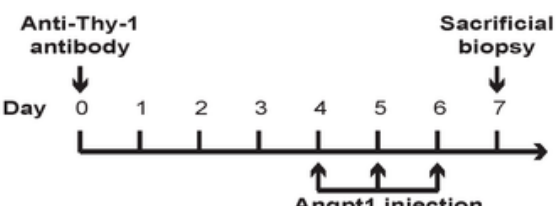

Figure 7
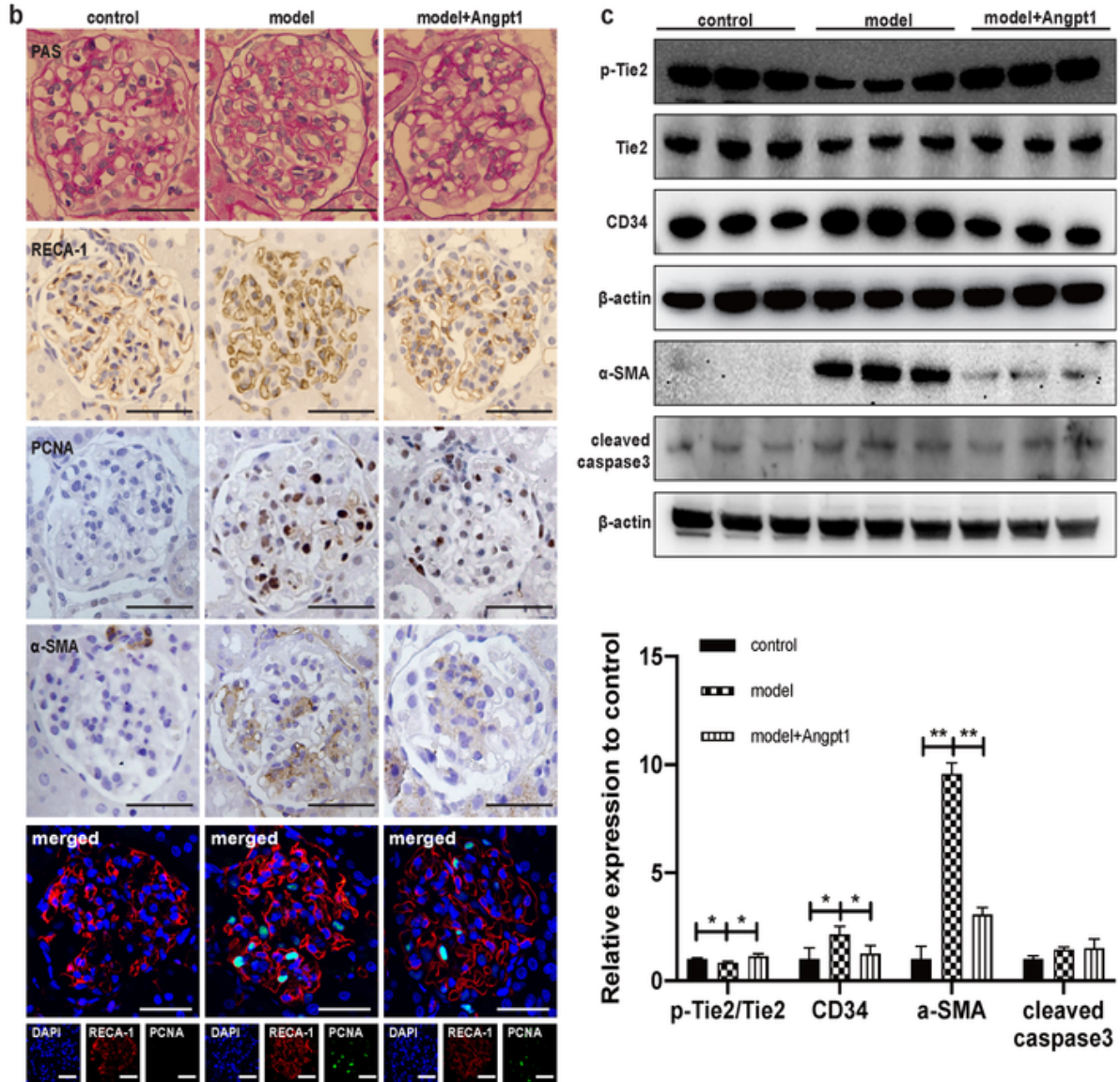

d
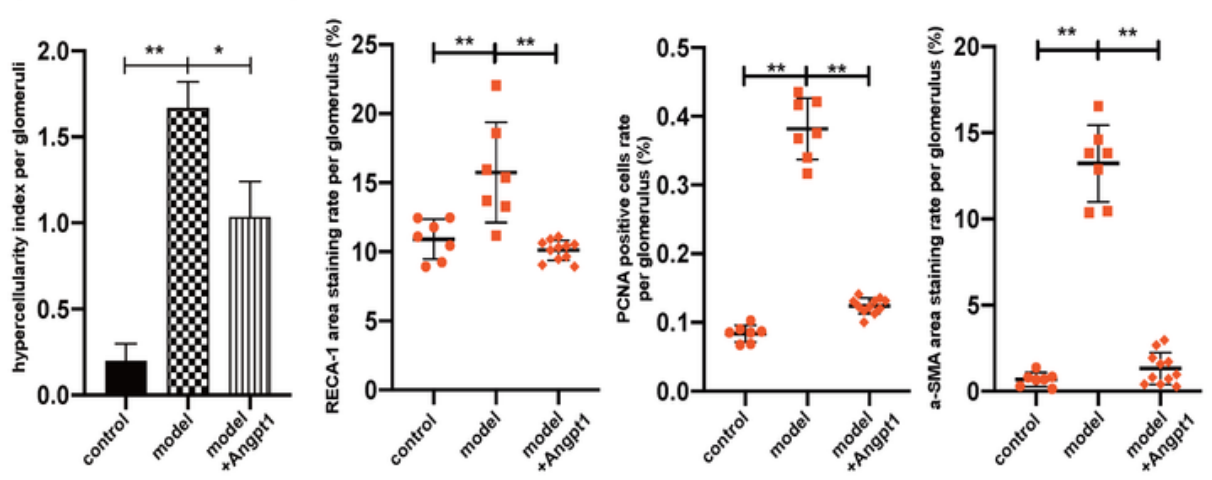

\section{Figure 7}

Angpt1 mimic peptide can effectively alleviate cell proliferation on the 7th day of anti-Thy-1 nephritis. (a) The rats were divided into the control group, model group and Angpt1-treated group. All rats were sacrificed on the 7th day after the model was established. (b and d) PAS staining results showed that the hypercellularity index per glomerulus decreased significantly after the Angpt1 mimic peptide was treated. $\times 400$ original magnification. (b and e) Immunohistochemical staining results showed that the PCNA- 
positive cells and the expression of RECA-1 and a-SMA in the glomeruli was significantly decreased after Angpt1 mimic peptide treatment. In addition, immunofluorescence co-staining of PCNA and RECA-1 showed that the number of double-positive cells in the glomeruli of rats in the Angpt1-treated group was decreased. The results are presented as the mean values $( \pm S D)$, ${ }^{\star *} \mathrm{P}<0.01$, control vs model, model vs model+Angpt1. $\times 400$ original magnification. (c) Western blot results showed that after the treatment with Angpt1 mimic peptide, the expression of a-SMA and CD34 was decreased and the phosphorylation level of Tie2 was increased, while the expression of caspase 3 was not affected. The results are presented as the mean values $( \pm S D),{ }^{*} P<0.5$, control vs model, model vs model + Angpt $1, * * P<0.01$, control vs model, model vs model+Angpt1. Control, normal saline was injected intravenously; model, anti-Thy-1 antibody was injected intravenously; model+Angpt1, anti-Thy-1 antibody was injected intravenously and Angpt1 mimic peptide was injected Intraperitoneally on the 4th, 5th and 6th day after the model was established. 


\section{activated mesangial cell}

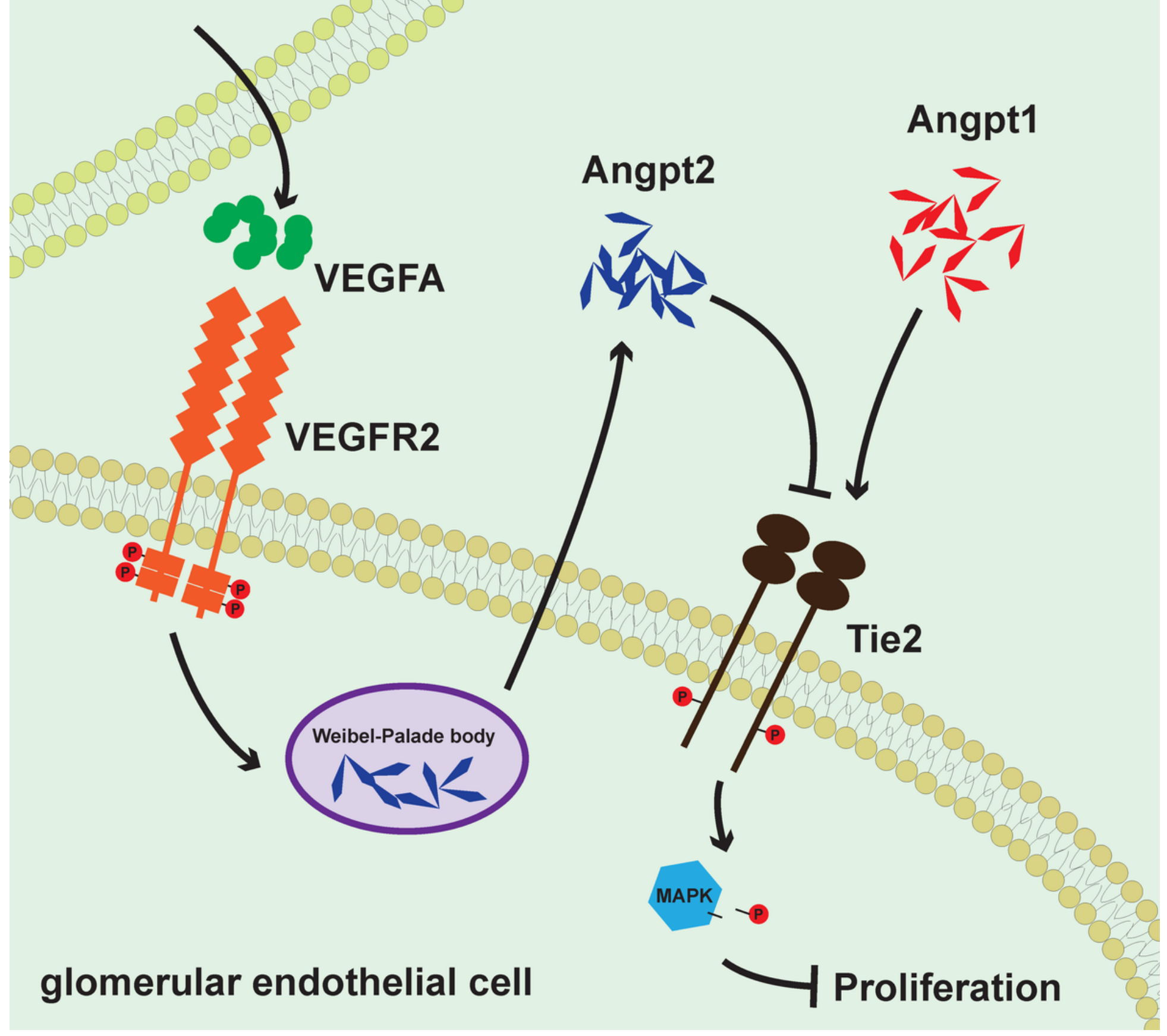

Figure 8

Activating MCs produce VEGFA which acts on VEGFR2, to promote ECs to release Angpt2. Angpt2 inhibits Tie2 phosphorylation and promotes ECs proliferation through MAPK pathway. Angpt1 mimic peptide can alleviate such process.

Supplementary Files 
This is a list of supplementary files associated with this preprint. Click to download.

- s2.pdf

- figureS1.pdf 